\title{
GEOMETRY OF 2-STEP NILPOTENT GROUPS WITH A LEFT INVARIANT METRIC. II
}

\author{
PATRICK EBERLEIN
}

\begin{abstract}
We obtain a partial description of the totally geodesic submanifolds of a 2-step, simply connected nilpotent Lie group with a left invariant metric. We consider only the case that $N$ is nonsingular; that is, ad $\xi: \mathscr{N} \rightarrow \mathcal{Z}$ is surjective for all elements $\xi \in \mathscr{N}-\mathscr{Z}$, where $\mathscr{N}$ denotes the Lie algebra of $N$ and $\mathscr{Z}$ denotes the center of $\mathscr{N}$. Among other results we show that if $H$ is a totally geodesic submanifold of $N$ with $\operatorname{dim} H \geq 1+\operatorname{dim} \mathscr{Z}$, then $H$ is an open subset of $g N^{*}$, where $g$ is an element of $H$ and $N^{*}$ is a totally geodesic subgroup of $N$. We find simple and useful criteria that are necessary and sufficient for a subalgebra $\mathscr{N}^{*}$ of $\mathscr{N}$ to be the Lie algebra of a totally geodesic subgroup $N^{*}$. We define and study the properties of a Gauss map of a totally geodesic submanifold $H$ of $N$. We conclude with a characterization of 2-step nilpotent Lie groups $N$ of Heisenberg type in terms of the abundance of totally geodesic submanifolds of $N$.
\end{abstract}

\section{INTRODUCTION}

Let $N$ denote a simply-connected, 2-step nilpotent Lie group with a left invariant metric $\langle$,$\rangle , and let \mathcal{N}=T_{e} N$ denote the Lie algebra of $N$. Let $\mathscr{Z}$ denote the center of $\mathscr{N}$ and $\mathscr{V}$ the orthogonal complement of $\mathscr{Z}$ in $\mathscr{N}$. The geometry of $N$ is determined by the properties of the linear map $j: \mathscr{Z} \rightarrow s o(\mathscr{V})$ defined by $\langle[X, Y], Z\rangle=\langle j(Z) X, Y\rangle$ for all $X, Y \in \mathscr{V}$ and $Z \in \mathscr{Z}$; see [E, K1, 2].

In [E] we studied geodesic behavior in $N$ and its quotient manifolds $\Gamma \backslash N$, where $\Gamma$ is a discrete cocompact subgroup of $N$. In this sequel we attempt to describe the totally geodesic submanifolds of $N$. We consider only the case that $\mathscr{N}$ is nonsingular; that is, the $\operatorname{map}$ ad $\xi: \mathscr{N} \rightarrow \mathscr{Z}$ is surjective for all elements $\xi \in \mathscr{N}-\mathscr{Z}$. The simplest examples of totally geodesic submanifolds of $N$ are the open subsets of $g N^{*}$, where $N^{*}$ is a totally geodesic subgroup of $N$ and $g$ is an arbitrary element of $N$. Our efforts here are directed mainly at the converse problem:

Under what conditions is a totally geodesic submanifold $H$ of

(*) $\quad N$ an open subset of $g N^{*}$ for some totally geodesic subgroup $N^{*}$ of $N$ and some element $g$ of $N$ ?

Received by the editors December 4, 1992.

1991 Mathematics Subject Classification. Primary 53C30; Secondary 22E25.

Key words and phrases. 2-step nilpotent Lie group, left invariant metric, totally geodesic subgroup, totally geodesic submanifold, Gauss map, Heisenberg type.

This research was supported in part by NSF Grant DMS-8901341. 
If $H$ is a 1-dimensional totally geodesic submanifold (i.e. a geodesic), then $H$ in general does not arise as in $(*)$, but the answer is unknown if $\operatorname{dim} H \geq 2$ and $N$ is nonsingular. In Corollary 5.6 we show that $H$ is an open subset of $g N^{*}$ as above if $\operatorname{dim} H \geq 1+\operatorname{dim} \mathscr{Z}$ and $N$ is nonsingular. From this fact it follows that $N$ admits no totally geodesic hypersurfaces.

Corollary 5.6 is a special case of something more general that we now describe. Let $\mathscr{V}, \mathscr{Z}$ also denote the left invariant distributions in $N$ determined by the subspaces of $\mathcal{N}=T_{e} N$. We say that a totally geodesic submanifold $H$ of $N$ is well positioned at a point $g$ of $H$ if $T_{g} H=\left\{T_{g} H \cap \mathscr{V}(g)\right\} \oplus\left\{T_{g} H \cap\right.$ $\mathscr{Z}(g)\}$. In Theorem 5.5 we show that if $N$ is nonsingular and $H$ is a totally geodesic submanifold of $N$ that is well positioned at every point of an open subset of $H$, then $H$ is an open subset of $g N^{*}$, where $g$ is an element of $H$ and $N^{*}$ is a totally geodesic subgroup of $N$. From Lemma 2.2 it follows that $H$ is well positioned at every point if $\operatorname{dim} H \geq 1+\operatorname{dim} \mathscr{Z}$, and Corollary 5.6 now follows immediately.

If $N$ is of Heisenberg type, a model space for the class of 2-step nilpotent groups with a left invariant metric (see $\S 1$ ), then every geodesic $\gamma$ of $N$ that begins at the identity $e$ is contained in a 3-dimensional totally geodesic subgroup $N^{*}$. Moreover, $N^{*}$ is isometric to the standard Heisenberg group after multiplying the metric of $N^{*}$ by a suitable positive constant that depends on the geodesic $\gamma$. The second main result of this paper is the following converse of the fact just stated: (Theorem 6.1). Let $N$ be a simply-connected, nonsingular, 2-step nilpotent Lie group with a left invariant metric. Assume that for every geodesic $\gamma$ of $N$ with $\gamma(0)=e$ there exists a connected, 3-dimensional, totally geodesic submanifold $H$ such that $\gamma^{\prime}(0) \in \mathscr{H}=T_{e} H$ and $\mathscr{C} \cap \mathscr{Z} \neq\{0\}$. Then $N$ is of Heisenberg type.

In the result above the hypothesis that $\mathscr{H} \cap \mathscr{Z}$ be nonzero is probably unnecessary. If $\operatorname{dim} \mathscr{Z}=1$ and $\operatorname{dim} H \geq 2$, then we show in Theorem 6.2 that $\mathscr{H} \cap \mathscr{Z} \neq\{0\}$ unless $\mathscr{H}$ is an abelian subspace of $\mathscr{V}$. (In this case $H$ is an open subset of the flat, totally geodesic subgroup $H^{*}=\exp (\mathscr{H})$.)

We now describe the organization and some of the main ideas of the paper. In $\S 1$ we provide definitions, examples and statements of some results from [E]. In $\S 2$ we discuss some applications of the Gauss submanifold equations, which provide necessary conditions for the existence of totally geodesic submanifolds of $N$. A systematic use of the Gauss equations that exploits the special nature of the curvature tensor of $N$ is one of the main tools of this paper. In $\S 3$ we obtain some useful results about totally geodesic subgroups of $N$ and the corresponding totally geodesic subalgebras of $\mathscr{N}$. In particular, Proposition 3.7 shows that a subalgebra $\mathscr{H}$ of $\mathscr{N}$ is totally geodesic if and only if one of the following three cases arises: (1) $\mathscr{H}$ is an abelian subspace of $\mathscr{V}$. (2) $\mathscr{H}$ is a subspace of $\mathscr{Z}$. (3) $\mathscr{H}$ is a nontrivial direct sum $\mathscr{H}=(\mathscr{H} \cap \mathscr{Z}) \oplus(\mathscr{H} \cap \mathscr{V})$ and $j(Z)$ leaves invariant $\mathscr{H} \cap \mathscr{V}$ for all $Z \in \mathscr{H} \cap \mathscr{Z}$. In cases (1) and (2) the corresponding totally geodesic subgroup $\bar{H}=\exp (\mathscr{H})$ is flat. In case (3) $\mathscr{H}$ is nonabelian and $\bar{H}$ is nonflat.

In $\S 4$ we study the Gauss map of a totally geodesic submanifold $H$ of $N$. If $H, N$ have dimensions $k, n$ respectively, then $G: H \rightarrow G(k, n)$ is defined by $G(g)=d L_{g-1}\left(T_{g} H\right)$ for all $g \in H$. Here $G(k, n)$ denotes the Grassmann manifold of $k$-dimensional subspaces of $\mathscr{N}=T_{e} N$. If $H$ is an open subset of 
$g N^{*}$, where $N^{*}$ is a totally geodesic subgroup of $N$ and $g$ is an element of $N$, then $G: H \rightarrow G(k, n)$ is a constant map. The main result of $\S 4$, Theorem 4.2 , is that for any connected totally geodesic submanifold $H$ of $N$ either $G$ is nonsingular at every point of $H$ or $G$ is constant on $H$ and $H$ is an open subset of $g N^{*}$ as above.

In $\S 5$ we obtain sufficient criteria for a totally geodesic submanifold $H$ that contains the identity to be an open subset of a totally geodesic subgroup $N^{*}$. The main result is Theorem 5.5, which we stated earlier. In $\S 6$ we obtain characterizations of groups $N$ of Heisenberg type that we described above.

\section{Preliminaries}

(1.1) Definition. A Lie algebra $\mathscr{N}$ is 2-step nilpotent if $[\mathcal{N}, \mathcal{N}]$ is nonzero and lies in the center of $\mathscr{N}$.

We introduce some notation that will be used throughout this paper.

(1.2) Notation. Let $\mathscr{N}$ denote a 2-step nilpotent Lie algebra, and let $N$ denote the simply-connected, 2-step nilpotent Lie group with Lie algebra $\mathscr{N}$. Let $\langle$,$\rangle denote an inner product on \mathscr{N}$, and let $\{\mathscr{N},\langle\rangle$,$\} denote the correspond-$ ing metric Lie algebra. Let $\{N,\langle\rangle$,$\} denote the group N$ equipped with the corresponding left invariant inner product. Let $\mathscr{Z}$ denote the center of $\mathscr{N}$, and let $\mathscr{V}=\mathscr{Z}^{\perp}$ denote the orthogonal complement of $\mathscr{Z}$ in $\mathscr{N}$ relative to $\langle$,$\rangle . For each nonzero element of Z$ of $\mathscr{Z}$ we let $j(Z): \mathscr{V} \rightarrow \mathscr{V}$ denote the skew symmetric linear transformation that satisfies

$$
\langle[X, Y], Z\rangle=\langle j(Z) X, Y\rangle \quad \text { for all } X, Y \in \mathscr{V}
$$

(1.3) Definition. A 2-step nilpotent Lie algebra $\mathscr{N}$ is nonsingular if ad $X: \mathcal{N}$ $\rightarrow \mathscr{Z}$ is surjective for each $X \in \mathscr{N}-\mathscr{Z}$. A simply-connected, 2-step nilpotent Lie group $N$ is nonsingular if its Lie algebra $\mathscr{N}$ is nonsingular.

(1.4) Remark. Let $\{\mathscr{N},\langle\rangle$,$\} be a nonsingular, 2-step nilpotent metric Lie$ algebra. Then

(1) $[\mathcal{N}, \mathscr{N}]=\mathscr{Z}$,

(2) $j(Z): \mathscr{V} \rightarrow \mathscr{V}$ is nonsingular for any nonzero $Z$ in $\mathscr{Z}$,

(3) $\operatorname{dim} \mathscr{V} \geq 1+\operatorname{dim} \mathscr{Z}$.

The first assertion follows since $\mathscr{Z}=$ ad $X(\mathscr{N}) \subseteq[\mathcal{N}, \mathscr{N}]$ if $X \in \mathscr{N}-\mathscr{Z}$. The second assertion follows routinely from the definitions. To prove (3) we let $X$ be any nonzero vector of $\mathscr{V}$ and consider the subspace $\mathscr{V}_{X}$ of $\mathscr{V}$ given by $\mathscr{V}_{X}=\{j(Z) X: Z \in \mathscr{Z}\}$. The map $Z \rightarrow j(Z) X$ is an isomorphism of $\mathscr{Z}$ onto $\mathscr{V}_{X}$ by (2). Assertion (3) now follows since $X$ is orthogonal to $\mathscr{V}_{X}$ by the skew symmetry of $j(Z), Z \in \mathscr{Z}$.

It is routine to prove the converse of (1.4).

(1.5) Lemma. Let $\{\mathscr{N},\langle\rangle$,$\} be a 2-step nilpotent metric Lie algebra such that$ $j(Z): \mathscr{V} \rightarrow \mathscr{V}$ is nonsingular for any nonzero $Z$ in $\mathscr{Z}$. Then $\mathscr{N}$ is nonsingular.

(1.6) Definition. A 2-step nilpotent metric Lie algebra $\{\mathscr{N},\langle\rangle$,$\} is of Heisen-$ berg type if there exists a positive constant $\lambda$ such that $j(Z)^{2}=-\lambda|Z|^{2}$ Id for every $Z \in \mathscr{Z}$. 
(1.7) Example. The standard metric Heisenberg algebras and groups. For a fixed integer $n \geq 1$ let $\left\{X_{1}, Y_{1}, \ldots, X_{n}, Y_{n}, Z\right\}$ be a basis of a $(2 n+1)$ dimensional real vector space $\mathscr{N}$. Equip $\mathscr{N}$ with a Lie algebra structure defined by the bracket relations

$$
\begin{array}{ll}
{\left[X_{i}, Y_{i}\right]=-\left[Y_{i}, X_{i}\right]=Z} & \text { for } 1 \leq i \leq n, \\
{\left[X_{i}, Z\right]=\left[Y_{i}, Z\right]=0} & \text { for } 1 \leq i \leq n, \\
{\left[Y_{i}, Y_{j}\right]=\left[X_{i}, X_{j}\left[=\left[X_{i}, Y_{j}\right]=0\right.\right.} & \text { for } i \neq j .
\end{array}
$$

If $\langle$,$\rangle is the inner product on \mathcal{N}$ for which $\left\{X_{1}, Y_{1}, \ldots, X_{n}, Y_{n}, Z\right\}$ is an orthonormal basis of $\mathcal{N}$, then $\{\mathscr{N},\langle\rangle$,$\} is a 2-step nilpotent metric Lie$ algebra with 1-dimensional center spanned by $Z$. One computes easily that $j(Z)^{2}=-$ Id. The metric Lie algebra $\{\mathcal{N},\langle\rangle$,$\} is called the standard metric$ $(2 n+1)$-dimensional Heisenberg algebra. The corresponding simply connected metric Lie group $\{N,\langle\rangle$,$\} is called a standard metric (2 n+1)$-dimensional Heisenberg group.

Conversely, let $\{\mathscr{N},\langle\rangle$,$\} be a 2-step nilpotent Lie algebra of Heisenberg$ type with 1-dimensional center $\mathscr{Z}$. If one multiplies the inner product $\langle$,$\rangle by$ a suitable positive constant, then the resulting metric Lie algebra is isometric and Lie algebra isomorphic to a standard metric Heisenberg algebra of dimension $2 n+1$ for some positive integer $n$.

To verify the assertion above let $\lambda>0$ be the constant such that $j(Z)^{2}=$ $-\lambda|Z|^{2}$ Id for all $Z \in \mathscr{Z}$. Define the inner product $\langle,\rangle^{*}=\lambda\langle$,$\rangle on \mathscr{N}$ and let ||$^{*}$ and $j^{*}(Z)$ define respectively the norm and linear transformation from (1.2) determined by $\langle,\rangle^{*}$. It is routine to check that $j^{*}(Z)=j(Z)$ for all $Z \in \mathscr{Z}$ and hence $j^{*}(Z)^{2}=-\left(|Z|^{*}\right)^{2}$ Id for all $Z \in \mathscr{Z}$.

Let $Z^{*}$ be a vector in $\mathscr{Z}$ with $\left|Z^{*}\right|^{*}=1$. Since $j^{*}\left(Z^{*}\right)$ is a nonsingular, skew symmetric linear transformation we can decompose $\mathscr{V}$ into an orthogonal direct sum $\mathscr{V}=\mathscr{V}_{1} \oplus \cdots \oplus \mathscr{V}_{n}$ for some integer $n \geq 1$, where $\operatorname{dim} \mathscr{V}_{i}=2$ and $\mathscr{V}_{i}$ is invariant under $j^{*}\left(Z^{*}\right)$ for every $i$. If $X_{i}^{*}$ is a unit vector in $\mathscr{V}_{i}$, then $Y_{i}^{*}=j^{*}\left(Z^{*}\right) X_{i}^{*}$ is a vector in $\mathscr{V}_{i}$ orthogonal to $X_{i}^{*}$ and $\left|Y_{i}\right|^{*}=1$ since $j^{*}\left(Z^{*}\right)^{2}=-$ Id . Hence $\left\{X_{1}^{*}, Y_{1}^{*}, \ldots, X_{n}^{*}, Y_{n}^{*}, Z^{*}\right\}$ is an orthonormal basis of $\mathscr{N}$ relative to $\langle,\rangle^{*}$. It is straightforward to check that the linear isomorphism of $\mathscr{N}$ onto the Heisenberg algebra of dimension $2 n+1$ which sends $X_{i}^{*}$ to $X_{i}, Y_{i}^{*}$ to $Y_{i}$ and $Z^{*}$ to $Z$ is also an isometry and a Lie algebra isomorphism.

(1.8) Example. The standard 3-dimensional metric Heisenberg algebra and group. If $\{\mathscr{N},\langle\rangle$,$\} is a nonabelian, 3-dimensional nilpotent metric Lie algebra,$ then $\mathscr{N}$ has 1 -dimensional center $\mathscr{Z}$ and 2-dimensional orthogonal complement $\mathscr{V}$. It is easy to see that $\mathscr{N}$ is of Heisenberg type. Hence $\{\mathscr{N}, \lambda\langle\rangle$, is a standard 3-dimensional metric Heisenberg algebra for some $\lambda>0$ by the discussion in (1.7).

The 3-dimensional Heisenberg algebra is Lie algebra isomorphic to the set of real $3 \times 3$ upper triangular matrices with zeros on the diagonal, where the matrix bracket operation is the usual one: $[A, B]=A B-B A$ for $3 \times 3$ matrices $A, B$. The natural isomorphism maps the basis $X, Y, Z$ for $\mathscr{N}$ onto those upper triangular matrices with zero entries except for a 1 in positions 12, 23, 13 respectively. Relative to the $3 \times 3$ matrix realization of the 3 -dimensional Heisenberg algebra $\mathscr{N}$ the corresponding simply-connected nilpotent group $N$ 
is the set of real $3 \times 3$ upper triangular matrices with ones on the diagonal, where the group multiplication is the usual one for $3 \times 3$ matrices.

For other examples of Lie algebras of Heisenberg type see [K1, 2] and (1.3) of $[\mathrm{E}]$.

(1.9) Exponential map. We let exp: $\mathscr{N} \rightarrow N$ denote the Lie group exponential map. It is known that exp is a diffeomorphism since $N$ is simply connected and nilpotent. See for example [R, p. 6].

For 2-step nilpotent Lie algebras the Campbell-Baker-Hausdorff formula becomes

$$
\exp (X) \cdot \exp (Y)=\exp (X+Y+(1 / 2)[X, Y])
$$

(1.10) Covariant derivative (cf. $\S 2.1$ of $[E]$ ). We may identify an element of $\mathscr{N}$ with a left invariant vector field on $N$ since $T_{e} N$ may be identified with $\mathscr{N}$. If $\xi_{1}, \xi_{2}$ are left invariant vector fields on $N$, then $\nabla_{\xi_{1}} \xi_{2}$ is left invariant also. One has the following formulas:

(a) $\nabla_{X} Y=\frac{1}{2}[X, Y]$ for all $X, Y \in \mathscr{V}$,

(b) $\nabla_{X} Z=-\frac{1}{2} j(Z) X$ for all $X \in \mathscr{V}, Z \in \mathscr{Z}$,

(c) $\nabla_{Z} Z^{*}=0$ for all $Z, Z^{*} \in \mathscr{Z}$.

(1.11) Curvature tensor (cf. $\S 2.1$ of [E]). If $\xi_{1}, \xi_{2}, \xi_{3}$ are left invariant vector fields on $N$, then $R\left(\xi_{1}, \xi_{2}\right) \xi_{3}=-\nabla_{\left[\xi_{1}, \xi_{2}\right]} \xi_{3}+\nabla_{\xi_{1}}\left(\nabla_{\xi_{2}} \xi_{3}\right)-\nabla_{\xi_{2}}\left(\nabla_{\xi_{1}} \xi_{3}\right)$ is also left invariant. One computes

(a) $R(X, Y) X^{*}=\frac{1}{2} j([X, Y]) X^{*}-\frac{1}{4} j\left(\left[Y, X^{*}\right]\right) X+\frac{1}{4} j\left(\left[X, X^{*}\right]\right) Y$ for all $X, Y, X^{*} \in \mathscr{V}$,

$$
R(X, Y) X=\frac{3}{4} j([X, Y]) X \quad \text { for all } X, Y \in \mathscr{V},
$$

(b) $R(X, Z) Z^{*}=-\frac{1}{4} j(Z) j\left(Z^{*}\right) X$ for all $X \in \mathscr{V}, Z, Z^{*} \in \mathscr{Z}$,

(c) $R(X, Z) Y=-\frac{1}{4}[X, j(Z) Y]$ for all $X, Y \in \mathscr{V}, Z \in \mathscr{Z}$,

(d) $R\left(Z_{1}, Z_{2}\right) Z_{3}=0$ for all $Z_{1}, Z_{2}, Z_{3} \in \mathscr{Z}$.

(1.12) Curvature transformations $R_{\xi}$. For each element $\xi$ of $\mathscr{N}$ let $\mathscr{R}_{\xi}$ be the curvature transformation defined by $R_{\xi}(\eta)=R(\eta, \xi) \xi$. The transformation $R_{\xi}$ is symmetric for each $\xi \in \mathscr{N}$ by the Bianchi identities for the Riemann curvature tensor. The next result is useful.

Lemma A. Let $\{\mathscr{N},\langle\rangle$,$\} be a nonsingular, 2-step nilpotent metric Lie algebra.$ Then

(a) If $\xi \in \mathscr{V}$ is nonzero, then $\mathscr{V}$ and $\mathscr{Z}$ are invariant subspaces for $R_{\xi}$. Moreover $R_{\xi}$ is negative semidefinite on $\mathscr{V}$ and positive definite on $\mathscr{Z}$.

(b) If $\xi \in \mathscr{Z}$ is nonzero, then $\mathscr{V}$ and $\mathscr{Z}$ are invariant subspaces for $R_{\xi}$. Moreover $R_{\xi}$ is positive definite on $\mathscr{V}$ and zero on $\mathscr{Z}$.

(c) Let $\xi$ lie in $\mathscr{V}$ or $\mathscr{Z}$. If $\eta$ is an eigenvector of $R_{\xi}$, then $\eta$ lies in $\mathscr{V}$ or $\mathscr{Z}$.

Proof. Let $\xi=X \in \mathscr{V}$ be nonzero. By (1.11) we see that

$$
R_{X}(Y)=-\frac{3}{4} j([X, Y]) X \in \mathscr{V} \quad \text { and } \quad\left\langle R_{X}(Y), Y\right\rangle=-\frac{3}{4}|[X, Y]|^{2} \leq 0 \text {. }
$$

If $Z$ is a nonzero element of $\mathscr{Z}$ then by (1.11) $R_{X}(Z)=\frac{1}{4}[X, j(Z) X] \in \mathscr{Z}$ and $\left\langle R_{X}(Z), Z\right\rangle=\frac{1}{4}|j(Z) X|^{2}>0$ since $j(Z)$ is nonsingular. This completes the proof of (a), and we omit the proof of (b), which is similar. Assertion (c) follows immediately from (a) and (b). 
The next result gives a description of Lie algebras of Heisenberg type in terms of curvature transformations.

Lemma B. Let $\{\mathscr{N},\langle\rangle$,$\} be a nonsingular, 2-step nilpotent metric Lie algebra.$ Then

(a) $\{\mathscr{N},\langle\rangle$,$\} is of Heisenberg type if and only if there exists a positive con-$ stant $\mu$ such that the restriction of $R_{Z}$ to $\mathscr{V}$ equals $\mu|Z|^{2}$ Id for all $Z \in \mathscr{Z}$.

(b) If $\{\mathscr{N},\langle\rangle$,$\} is of Heisenberg type with j(Z)^{2}=-\lambda|Z|^{2}$ Id for some positive constant $\lambda$ and all $Z \in \mathscr{Z}$, then $[X, j(Z) X]=\lambda|X|^{2} Z$ for all $X \in \mathscr{V}$ and all $Z \in \mathscr{Z}$. In particular the restriction of $R_{X}$ to $\mathscr{Z}$ equals $\frac{1}{4} \lambda|X|^{2}$ Id for every $X \in \mathscr{V}$.

Proof. Assertion (a) follows from (1.11) since the restriction of $R_{Z}$ to $\mathscr{V}$ equals $-\frac{1}{4} j(Z)^{2}$ for all $Z \in \mathscr{Z}$. Now let $\{\mathscr{N},\langle\rangle$,$\} be a 2-step nilpotent,$ metric Lie algebra of Heisenberg type, and let $\lambda$ be a positive constant such that $j(Z)^{2}=-\lambda|Z|^{2}$ Id on $\mathscr{V}$ for all $Z \in \mathscr{Z}$. If $X \in \mathscr{V}$ and $Z \in \mathscr{Z}$ are arbitrary elements, then $[X, j(Z) X]=\lambda|X|^{2} Z$ by the lemma in Example 4 of (2.11) of [E]. Hence $R_{X}(Z)=\frac{1}{4}[X, j(Z) X]=\frac{\lambda}{4}|X|^{2} Z$ by (1.11), which completes the proof of $(b)$.

\section{Using the Gauss equations}

If $X^{*}$ is a totally geodesic submanifold of a Riemannian manifold $X$, then the Gauss submanifold equations imply that the curvature tensor of $X^{*}$ is the restriction of the curvature tensor of $X$ to the tangent spaces of $X^{*}$. In the present case we obtain

(2.1) Lemma. Let $\{N,\langle\rangle$,$\} be a simply-connected, nonsingular, 2-step nilpo-$ tent Lie group with a left invariant metric. Let $H$ be a totally geodesic submanifold of $N$ that contains the identity, and let $\mathscr{H}=T_{e} H \subseteq T_{e} N=\mathscr{N}$. If $\xi_{1}$, $\xi_{2}, \xi_{3}$ are arbitrary elements of $\mathscr{H}$ then $R\left(\xi_{1}, \xi_{2}\right) \xi_{3} \in \mathscr{H}$, where $R$ is the curvature tensor of $\mathcal{N}$.

We will use the result above frequently. We note that when studying totally geodesic submanifolds of $N$ it suffices to consider those that contain the identity since the left translations by elements of $N$ form a transitive subgroup of isometries of $N$.

We give a useful application of the result above.

(2.2) Lemma. Let $H$ be a totally geodesic submanifold of $N$ that contains the identity $e$, and let $\mathscr{H}=T_{e} H \subseteq \mathscr{N}$. If $\mathscr{H} \cap \mathscr{V} \neq 0$ or $\mathscr{H} \cap \mathscr{Z} \neq 0$, then

(a) $\mathscr{H}=(\mathscr{H} \cap \mathscr{V}) \oplus(\mathscr{H} \cap \mathscr{Z})$ (orthogonal direct sum),

(b) $\mathscr{H} \cap \mathscr{V}$ is invariant under $j(Z)^{2}$ for every $Z \in \mathscr{H} \cap \mathscr{Z}$,

(c) $[X, j(Z) Y] \in \mathscr{H} \cap \mathscr{Z}$ for any elements $X, Y \in \mathscr{H} \cap \mathscr{V}$ and $Z \in \mathscr{H} \cap \mathscr{Z}$,

(d) If $j(Z)$ leaves invariant $\mathscr{H} \cap \mathscr{V}$ for some nonzero element $Z$ of $\mathscr{H} \cap \mathscr{Z}$, then $\mathscr{H}$ is a totally geodesic subalgebra of $\mathscr{N}$. (See (3.2) below.)

Proof. (a) Let $\xi$ be a nonzero element of $\mathscr{H} \cap \mathscr{V}$ or $\mathscr{H} \cap \mathscr{Z}$ and consider the curvature transformation $R_{\xi}$ given by $R_{\xi}\left(\xi^{*}\right)=R\left(\xi^{*}, \xi\right) \xi$. By (2.1) $R_{\xi}$ leaves $\mathscr{H}$ invariant and by (a), (b) of Lemma A in (1.12) $R_{\xi}$ leaves both $\mathscr{H} \cap \mathscr{V}$ and $\mathscr{H} \cap \mathscr{Z}$ invariant. Since $R_{\xi}$ is symmetric there exists an orthonormal basis of $\mathscr{H}$ that consists of eigenvalues of $R_{\xi}$. By (c) of (1.12) each eigenvector of $R_{\xi}$ in $\mathscr{H}$ must lie in $\mathscr{H} \cap \mathscr{V}$ or $\mathscr{H} \cap \mathscr{Z}$. This proves (a). 
(b) If $Z$ is any element of $\mathscr{H} \cap \mathscr{Z}$, then $j(Z)^{2}$ is the restriction of $-4 R_{Z}$ to $\mathscr{V}$ by (1.11). The assertion follows since $R_{Z}$ leaves $\mathscr{H} \cap \mathscr{V}$ invariant by (b) of (1.12) and (2.1).

(c) If $X, Y \in \mathscr{H} \cap \mathscr{V}$ and $Z \in \mathscr{H} \cap \mathscr{Z}$ are given arbitrarily, then by (1.11) and (2.1) $\mathscr{H}$ contains $-4 R(X, Z) Y=[X, j(Z) Y]$.

(d) We may assume that $\mathscr{H} \cap \mathscr{V} \neq\{0\}$ for otherwise $\mathscr{H}$ is a totally geodesic subalgebra of $\mathscr{Z}$ by (a) and (1.10) (see (3.2) below for the definition of a totally geodesic subalgebra). Given elements $X, Y$ of $\mathscr{H} \cap \mathscr{V}$ let $Y^{*}=j(Z)^{-1} Y \in$ $\mathscr{H} \cap \mathscr{V}$. Then $[X, Y]=\left[X, j(Z) Y^{*}\right] \in \mathscr{H} \cap \mathscr{Z}$ by (c), and from (a) it follows that $[\mathscr{H}, \mathscr{H}]=[\mathscr{H} \cap \mathscr{V}, \mathscr{H} \cap \mathscr{V}] \subseteq \mathscr{H}$. Hence $\mathscr{H}$ is a subalgebra.

Let elements $X^{*} \in \mathscr{H} \cap \mathscr{V}$ and $Z^{*} \in \mathscr{H} \cap \mathscr{Z}$ be given. To prove that $\mathscr{H}$ is totally geodesic it suffices by (3.7) below to prove that $j\left(Z^{*}\right) X^{*} \in \mathscr{H}$. If $X^{\prime}=$ $j(Z)^{-1} X^{*} \in \mathscr{H} \cap \mathscr{V}$, then by (1.11) and (2.1) $\mathscr{H}$ contains $-4 R\left(X^{\prime}, Z^{*}\right) Z=$ $j\left(Z^{*}\right) j(Z) X^{\prime}=j\left(Z^{*}\right) X^{*}$.

\section{TOTALly GEODESIC SUbALGEBRAS AND SUbGROUPS}

Let $\{N,\langle\rangle$,$\} be a simply-connected, nonsingular, 2-step nilpotent Lie group$ with a left invariant metric. Let $\mathcal{N}$ denote the Lie algebra of $N$.

(3.1) Definition. A totally geodesic subgroup of $N$ is a connected Lie subgroup $N^{*}$ such that $N^{*}$ is totally geodesic as a Riemannian submanifold of $N$.

(3.2) Definition. A subalgebra $\mathscr{N}^{*}$ of $\mathscr{N}$ is totally geodesic if $\nabla_{\xi_{1}} \xi_{2} \in \mathscr{N}^{*}$ whenever $\xi_{1}, \xi_{2} \in \mathscr{N}^{*}$.

(3.3) Definition. A subalgebra $\mathscr{N}^{*}$ of $\mathscr{N}$ is flat if the corresponding Lie subgroup $N^{*}=\exp \left(\mathscr{N}^{*}\right)$ is flat as a Riemannian submanifold of $N$.

It is routine to check that a connected Lie subgroup $N^{*}$ is totally geodesic if and only if its Lie algebra $\mathscr{N}^{*}$ is totally geodesic. If $N^{*}$ is a totally geodesic subgroup with Lie algebra $\mathscr{N}^{*}=T_{e} N^{*}$, then $N^{*}$ is closed as a subset of $N$ and hence complete as a Riemannian manifold since $N^{*}=\exp \left(\mathscr{N}^{*}\right)$ and exp: $\mathscr{N} \rightarrow N$ is a diffeomorphism.

(3.4) Examples. (1) Let $\mathscr{H}$ be a vector subspace of $\mathscr{Z}$. Then $\mathscr{H}$ is an abelian, flat, totally geodesic subalgebra of $\mathscr{N}$ by (1.10) and (1.11). The group $H=\exp (\mathscr{H})$ is an abelian, flat, totally geodesic subgroup of $N$.

(2) Let $\mathscr{H}$ be an abelian subspace of $\mathscr{V}$; that is, $[X, Y]=0$ for all $X$, $Y \in \mathscr{H}$. Then $\mathscr{H}$ is an abelian, flat, totally geodesic subalgebra of $\mathscr{N}$ by (1.10) and (1.11). The group $H=\exp (H)$ is an abelian, flat, totally geodesic subgroup of $N$.

If $\operatorname{dim} \mathscr{V} \geq 2+\operatorname{dim} \mathscr{Z}$, then every nonzero element $X$ of $\mathscr{V}$ lies in an abelian subspace of $\mathscr{V}$ of dimension at least 2 since ad $X: \mathscr{V} \rightarrow \mathscr{Z}$ is surjective; recall that $\operatorname{dim} \mathscr{V} \geq 1+\operatorname{dim} \mathscr{Z}$ by (3) of (1.4). More generally, if $\operatorname{dim} \mathscr{V} \geq 1+r+r \operatorname{dim} \mathscr{Z}$ for some integer $r \geq 2$, then every nonzero element $X$ of $\mathscr{V}$ lies in an abelian subspace of $\mathscr{V}$ of dimension $r+1$. See (2.11) of [E] for details.

We shall see below in (3.6) that the examples (1) and (2) above are the only examples of flat, totally geodesic subalgebras of $\mathscr{N}$.

(3) Let $\{\mathscr{N},\langle\rangle$,$\} be a 2-step, nilpotent metric Lie algebra of Heisenberg$ type. If $X \in \mathscr{V}$ and $Z \in \mathscr{Z}$ are arbitrary nonzero vectors, then $\mathscr{H}=$ 
$\operatorname{span}\{X, Z, j(Z) X\}$ is a 3-dimensional totally geodesic subalgebra of $\mathscr{N}$. If the metric $\langle$,$\rangle on \mathscr{H}$ is multiplied by a suitable positive constant $\lambda$, then the subgroup $H=\exp (\mathscr{H})$ is isometric to the standard metric 3-dimensional Heisenberg group. See (1.7) above and (2.11) of [E] for further details.

Next we consider some necessary and sufficient criteria for a subalgebra $\mathscr{H}$ of $\mathcal{N}$ to be totally geodesic.

(3.5) Proposition. Let $\{N,\langle\rangle$,$\} be a simply-connected, nonsingular, 2-step$ nilpotent Lie group with a left invariant metric. Let $\mathscr{H}$ be a totally geodesic subalgebra of $\mathscr{N}$. Then exactly one of the following occurs:

(1) $\mathscr{H}$ is an abelian subspace of $\mathscr{V}$ and $\mathscr{H}$ is flat.

(2) $\mathscr{H}$ is a subspace of $\mathscr{Z}$ and $\mathscr{H}$ is flat.

(3) (a) $\mathscr{H}$ is the direct sum of the nonzero subspaces $\mathscr{H} \cap \mathscr{V}$ and $\mathscr{H} \cap \mathscr{Z}$, (b) $\mathscr{H} \cap \mathscr{V}$ is invariant under $j(Z)$ for every $Z \in \mathscr{H} \cap \mathscr{Z}$.

Remark. Compare (3) above with Lemma (2.2).

(3.6) Corollary. If $\mathscr{H}$ is an abelian, totally geodesic subalgebra of $\mathscr{N}$, then either $\mathscr{H} \subseteq \mathscr{Z}$ or $\mathscr{H}$ is an abelian subspace of $\mathscr{V}$.

For the moment we assume the result of (3.5) and prove the corollary. It suffices to show that if $\mathscr{H}$ satisfies case (3) of (3.5), then $\mathscr{H}$ is nonabelian. Let $\mathscr{H}$ be a totally geodesic subalgebra of $\mathscr{N}$ that satisfies case (3) of (3.5), and let $X \in \mathscr{H} \cap \mathscr{V}$ and $Z \in \mathscr{H} \cap \mathscr{Z}$ be arbitrary nonzero elements. By (b) of (3.5) $\mathscr{H}$ contains $j(Z) X$ and hence $\mathscr{H}$ contains $[X, j(Z) X]$ since $\mathscr{H}$ is a subalgebra. However, $[X, j(Z) X]$ is nonzero since $\langle[X, j(Z) X], Z\rangle=|j(Z) X|^{2}>0$, and this shows that $\mathscr{H}$ is nonabelian.

We now prove (3.5). Let $\mathscr{H}$ be a totally geodesic subalgebra of $\mathscr{N}$, and let $\xi$ be an element of $\mathscr{H}$. Write $\xi=X+Z$, where $X \in \mathscr{V}$ and $Z \in \mathscr{Z}$. By (3.2) $\mathscr{H}$ contains $\nabla_{\xi} \xi=-j(Z) X$, and hence $\mathscr{H}$ also contains $[\xi, j(Z) X]=$ $[X, j(Z) X]$. We consider separately two cases: (1) $[X, j(Z) X] \neq 0$ for some element $\xi=X+Z \in \mathscr{H}$. (2) $[X, j(Z) X]=0$ for every element $\xi=X+Z \in$ $\mathscr{H}$.

If case (1) occurs, then $\mathscr{H}$ is nonabelian and $\mathscr{H} \cap \mathscr{Z} \neq\{0\}$. By (2.2) $\mathscr{H}=\mathscr{H} \cap \mathscr{V} \oplus \mathscr{H} \cap \mathscr{Z}$, and $\mathscr{H} \cap \mathscr{V} \neq\{0\}$ since $\mathscr{H}$ is nonabelian. If $X \in \mathscr{H} \cap \mathscr{V}$ and $Z \in \mathscr{H} \cap \mathscr{Z}$ are arbitrary vectors, then by (3.2) $\mathscr{H}$ contains $\nabla_{X} Z=-\frac{1}{2} j(Z) X$. Hence case (3) of (3.5) occurs.

Suppose now that $[X, j(Z) X]=0$ for every element $\xi=X+Z \in \mathscr{H}$. Then $0=\langle[X, j(Z) X], Z\rangle=|j(Z) X|^{2}$ for every element $\xi=X+Z \in \mathscr{H}$. We conclude that either $X=0$ or $Z=0$ for every element $\xi=X+Z \in \mathscr{H}$ since $j(Z)$ is nonsingular if $Z \neq 0$ by (1.4). It follows that either $\mathscr{H} \subseteq \mathscr{V}$ or $\mathscr{H} \subseteq \mathscr{Z}$ since $\mathscr{H}$ cannot be the set union of the subspaces $\mathscr{V}$ and $\mathscr{Z}$ if both subspaces are nonzero. If $\mathscr{H} \subseteq \mathscr{Z}$ then $\mathscr{H}$ is clearly abelian and flat by (1.11). If $\mathscr{H} \subseteq \mathscr{V}$ then $[\mathscr{H}, \mathscr{H}] \subseteq \mathscr{H} \cap \mathscr{Z}=\{0\}$ since $\mathscr{H}$ is a subalgebra, and hence $\mathscr{H}$ is abelian in this case also. By (1.11) $\mathscr{H}$ is flat.

We now derive a converse of (3.5).

(3.7) Proposition. Let $\mathscr{H}$ be a subalgebra of $\mathscr{N}$ that satisfies one of the three conditions of (3.5). Then $\mathscr{H}$ is totally geodesic.

Proof. If $\mathscr{H}$ is an abelian subalgebra of $\mathscr{V}$ or $\mathscr{Z}$ then $\mathscr{H}$ is totally geodesic by (1.10). Suppose now that $\mathscr{H}$ satisfies case (3) of (3.5). Let $\xi_{1}, \xi_{2}$ be 
arbitrary elements of $\mathscr{H}$ and write $\xi_{i}=X_{i}+Z_{i}$, where $X_{i} \in \mathscr{V}$ and $Z_{i} \in \mathscr{Z}$ for $i=1,2$. By $3(\mathrm{a})$ of (3.5) we see that $X_{i} \in \mathscr{H} \cap \mathscr{V}$ and $Z_{i} \in \mathscr{H} \cap \mathscr{Z}$ for $i=1,2$. By $3(\mathrm{~b}),(1.10)$ and the fact that $\mathscr{H}$ is a subalgebra of $\mathscr{N}$ we see that $\mathscr{H}$ contains $\frac{1}{2}\left[X_{1}, X_{2}\right]-\frac{1}{2} j\left(Z_{2}\right) X_{1}-\frac{1}{2} j\left(Z_{1}\right) X_{2}=\nabla_{\xi_{1}} \xi_{2}$. Hence $\mathscr{H}$ is totally geodesic.

The next result will be useful later.

(3.8) Corollary. Let $X, Z$ be nonzero elements of $\mathscr{V}, \mathscr{Z}$ respectively such that

(a) $[X, j(Z) X]=\lambda Z$ for some $\lambda \in \mathbb{R}$,

(b) $j(Z)^{2} X=\mu X$ for some $\mu \in \mathbb{R}$.

Then $\mathscr{H}=\operatorname{span}\{X, j(Z) X, Z\}$ is a nonabelian totally geodesic subalgebra of $\mathscr{N}$.

Proof. The vector subspace $\mathscr{H}$ is a subalgebra by (a). From (b) we see that $\mathscr{H} \cap \mathscr{V}=\operatorname{span}\{X, j(Z) X\}$ is invariant under $j(Z)$. Since $Z$ spans $\mathscr{H} \cap \mathscr{Z}$ it follows from (3.7) that $\mathscr{H}$ is totally geodesic. The subalgebra $\mathscr{H}$ is nonabelian by (a) since $\lambda|Z|^{2}=\langle[X, j(Z) X], Z\rangle=|j(Z) X|^{2}>0$, which implies that $\lambda>0$.

We conclude this section with some elementary results that will be useful later.

(3.9) Lemma. Let $X$ be a Riemannian manifold, and let $H_{1}, H_{2}$ be totally geodesic submanifolds that contain a point $x^{*}$. Let $V=T_{x^{*}} H_{1} \cap T_{x^{*}} H_{2}$ and suppose that $\operatorname{dim} V=k \geq 1$. Then there exists a positive number $\varepsilon$ such that $B_{\varepsilon}\left(x^{*}\right) \cap H_{1} \cap H_{2}$ contains an arc connected, $k$-dimensional, totally geodesic submanifold $H_{\varepsilon}$ of $X$, where $B_{\varepsilon}\left(x^{*}\right)$ denotes the open metric ball in $X$ of center $x^{*}$ and radius $\varepsilon$. Moreover, $T_{x} H_{\varepsilon}=T_{x} H_{1} \cap T_{x} H_{2}$ for all $x \in H_{\varepsilon}$.

(3.10) Corollary. Let $X$ be a Riemannian manifold and let $H_{1}, H_{2}$ be totally geodesic submanifolds that contain a point $x^{*}$. Let $H_{1}, H_{2}$ be connected and let $H_{2}$ be closed as a subset of $X$. If $T_{X^{*}} H_{1} \subset T_{x^{*}} H_{2}$, then $H_{1} \subseteq H_{2}$. If $T_{x^{*}} H_{1}=T_{x^{*}} H_{2}$, then $H_{1}$ is an open subset of $H_{2}$. fact.

Before proving these two results we apply them to get the following useful

(3.11) Proposition. Let $\{N,\langle\rangle$,$\} be a simply-connected, nonsingular, 2-step$ nilpotent Lie group with a left invariant metric. Let $H$ be a totally geodesic submanifold of $N$ that contains the identity $e$, and let $\mathscr{H}=T_{e} H$. Then the following are equivalent:

(1) $\mathscr{H}$ is a totally geodesic subalgebra of $\mathscr{N}$,

(2) $\bar{H}=\exp (\mathscr{H})$ is a totally geodesic subgroup and $H$ is an open subset of $\bar{H}$.

We prove (3.11). Clearly (2) implies (1). If (1) holds, then $\bar{H}=\exp (\mathscr{H})$ is a totally geodesic subgroup, and $H$ is an open subset of $\bar{H}$ by (3.10).

We assume for the moment that (3.9) has been proved, and we prove (3.10). Let $A=\left\{x \in H_{1}: x \in H_{1} \cap H_{2}\right.$ and $\left.T_{x} H_{1} \subseteq T_{x} H_{2}\right\}$. Clearly $A$ is a closed subset of $H_{1}$ since $H_{2}$ is a closed subset of $X$. Moreover, $A$ is nonempty since it contains $x^{*}$ by hypothesis. By (3.9) $A$ is open in $H_{1}$, and hence $H_{1} \subseteq H_{2}$. 
If $T_{x^{*}} H_{1}=T_{x^{*}} H_{2}$ it follows that $H_{1}$ is an open subset of $H_{2}$ since $H_{1}, H_{2}$ have the same dimension.

We now prove (3.9). For a small positive number $\varepsilon$ let $B_{\varepsilon}(0)=\{v \in$ $\left.T_{x^{*}} X:|v|<\varepsilon\right\}$ and $B_{\varepsilon}\left(x^{*}\right)=\left\{x \in N: d\left(x, x^{*}\right)<\varepsilon\right\}$. Let $V_{\varepsilon}=B_{\varepsilon}(0) \cap T_{X^{*}} H_{1} \cap$ $T_{x^{*}} H_{2}$ and $H_{\varepsilon}=\operatorname{Exp}_{x^{*}}\left(V_{\varepsilon}\right) \subseteq B_{\varepsilon}\left(x^{*}\right) \cap H_{1} \cap H_{2}$, where $\operatorname{Exp}_{x^{*}}: T_{x^{*}} X \rightarrow X$ denotes the Riemannian exponential map of $X$. Clearly $H_{\varepsilon}$ is an arc connected submanifold of dimension $k=\operatorname{dim} V$. If $\varepsilon$ is sufficiently small and $O_{i}=\operatorname{Exp}_{x^{*}}\left(B_{\varepsilon}(0) \cap T_{x^{*}} H_{i}\right)$, then $O_{i}$ is an open subset of $H_{i}$ for $i=1,2$, $O_{1} \cap O_{2}=H_{\varepsilon}$ and $T_{x} H_{\varepsilon}=T_{x} H_{1} \cap T_{x} H_{2}$ for all $x \in H_{\varepsilon}$. Given $x \in H_{\varepsilon}$ and $v \in T_{x} H_{\varepsilon}=T_{x} H_{1} \cap T_{x} H_{2}$ it follows that $\operatorname{Exp}_{x}(t v) \in H_{\varepsilon}=O_{1} \cap O_{2}$ for small $t$ since $H_{1}, H_{2}$ are totally geodesic, and $O_{1}, O_{2}$ are open in $H_{1}, H_{2}$. We conclude that $H_{\varepsilon}$ is totally geodesic.

\section{GaUSS MAP OF A TOTALly GeODESIC SUBMANIFOLD}

Let $\{N,\langle\rangle$,$\} be a simply-connected, nonsingular, 2-step nilpotent Lie group$ of dimension $n \geq 3$ with a left invariant metric. Let $H$ be a totally geodesic submanifold of $N$, not necessarily complete, of dimension $k \geq 2$. Let $G(k, n)$ denote the Grassmann manifold of $k$-dimensional subspaces of $\mathscr{N}=T_{e} N$. We define a Gauss map $G: H \rightarrow G(k, n)$ by $G(g)=d L_{g^{-1}}\left(T_{g} H\right)=T_{e} H_{g}$, where $H_{g}=L_{g^{-1}}(H)$, a totally geodesic submanifold of dimension $k$ that contains the identity.

If $H$ is an open subset of $L_{g}(\bar{H})$ for some $g \in H$, where $\bar{H}$ is a totally geodesic subgroup of $N$, then it is easy to see from the definition that the Gauss map is constant on $H$. The main result of this section, Theorem (4.2), is that if $G: H \rightarrow G(k, n)$ fails to have maximal rank at some point $g$ of $H$, then $H$ is an open subset of $L_{g}(\bar{H})$, where $\bar{H}$ is a totally geodesic subgroup of $N$. It follows that either $G$ has maximal rank at every point $g$ of $H$ or $G$ is constant on $H$.

We use (4.2) to obtain in (4.3) some necessary and sufficient conditions for $\mathscr{H}=T_{e} H$ to be a totally geodesic subalgebra of $\mathscr{N}$, where $H$ is a totally geodesic submanifold of $N$ that contains the identity.

We begin by considering totally geodesic submanifolds $H$ that contain the identity.

(4.1) Proposition. Let $\{N,\langle\rangle$,$\} be a simply-connected, nonsingular, 2-step$ nilpotent Lie group of dimension $n \geq 3$ with a left invariant metric. Let $H$ be a totally geodesic submanifold of $N$ of dimension $k \geq 2$ that contains the identity, and let $\mathscr{H}=T_{e} H \subseteq \mathscr{N}$. let $G: H \rightarrow G(k, n)$ denote the Gauss map of $H$. Then $d G\left(\xi^{*}\right)=0$ for $\xi^{*} \in \mathscr{H}$ if and only if $\nabla_{\xi^{*}} \xi \in \mathscr{H}$ for every element $\xi \in \mathscr{H}$.

We shall need the following:

Lemma. Let $\xi, \xi^{*}$ be nonzero elements of $\mathscr{H}$, and let $\gamma(t)$ be the geodesic of $N$ such that $\gamma(0)=e$ and $\gamma^{\prime}(0)=\xi^{*}$. Let $P_{t}: T_{e} N \rightarrow T_{\gamma(t)} N$ denote parallel translation along $\gamma$. Let $\xi(t)=d L_{\gamma(t)^{-1}}\left(P_{t} \xi\right)$ for every $t$, and let $\mathscr{H}_{t}=G(\gamma t)=$ $d L_{\gamma(t)-1}\left(T_{\gamma(t)} H\right)$. Then

(a) $\xi(t) \in \mathscr{H}_{t}$ for every $t$, 
(b) $\xi(t)=\xi-t\left(\nabla_{\xi^{*}} \xi\right)+O\left(t^{2}\right)$ for every $t$, where $O\left(t^{2}\right)$ denotes a vector in $\mathcal{N}$ such that $\left|O\left(t^{2}\right)\right| \leq M|t|^{2}$ for some $M>0$ and all small $t$.

Proof of the lemma. We note that $\gamma(-\varepsilon, \varepsilon) \subseteq H$ for a small positive number $\varepsilon$ since $H$ is totally geodesic. In particular $\mathscr{H}_{t}$ makes sense for $|t|<\varepsilon$.

In the discussion below we sometimes identify an element $\xi$ of $\mathscr{N}=T_{e} N$ with the unique left invariant vector field on $N$ determined by $\xi$.

Let $V, W$ be $C^{\infty}$ vector fields in $N$ such that $V(e)=v \neq 0$. Let $\gamma(t)$ be the geodesic in $N$ with $\gamma(0)=e, \gamma^{\prime}(0)=v$, and let $P_{t}: T_{e} N \rightarrow T_{\gamma(t)} N$ denote parallel translation along $\gamma$. A straightforward power series expansion shows

$$
W(\gamma t)=P_{t}\left\{W(e)+t\left(\nabla_{V} W\right)(e)+O\left(t^{2}\right)\right\} .
$$

Let $\xi, \xi^{*}$ be nonzero left invariant vector fields on $N$ such that $\xi(e)$ and $\xi^{*}(e)$ lie in $\mathscr{H}=T_{e} H$. If $\gamma(t)$ is the geodesic in $N$ with $\gamma(0)=e, \gamma^{\prime}(0)=\xi^{*}$, then by applying (1) to the vector fields $V=\xi^{*}$ and $W=\xi, \nabla_{\xi^{*}} \xi$ we obtain

$$
P_{t} \xi(e)=\xi(\gamma t)-t\left(\nabla_{\xi^{*}} \xi\right)(\gamma t)+O\left(t^{2}\right) .
$$

Since $H$ is totally geodesic in $N$ and $\gamma^{\prime}(0) \in T_{e} H$ it follows that $\gamma(t) \in H$ and $P_{t} \xi(e) \in T_{\gamma(t)} H$ for $|t|<\varepsilon$, where $\varepsilon$ is small. From (2) we conclude that for $|t|<\varepsilon$ the vector space $\mathscr{H}_{t}=d L_{\gamma(t)-1}\left(T_{\gamma(t)} H\right)$ contains

$$
\xi(t)=d L_{\gamma(t)^{-1}}\left(P_{t} \xi(e)\right)=\xi(e)-t\left(\nabla_{\xi^{*}} \xi\right)(e)+O\left(t^{2}\right) .
$$

We prove Proposition (4.1). Let $\xi^{*}$ be an arbitrary nonzero element of $\mathscr{H}=T_{e} H$, and let $\gamma:(-\varepsilon, \varepsilon) \rightarrow H$ be the unique geodesic of $N$ and $H$ such that $\gamma(0)=e$ and $\gamma^{\prime}(0)=\xi^{*}$. If $G: H \rightarrow G(k, n)$ is the Gauss map of $H$, then $d G\left(\xi^{*}\right)=(G \circ \gamma)^{\prime}(0)$, where $G(\gamma t)=\mathscr{H}_{t}$ in the notation of the lemma above.

If $\varepsilon$ is sufficiently small, then there exists a uniquely determined $C^{\infty}$ curve $\varphi:(-\varepsilon, \varepsilon) \rightarrow \operatorname{Hom}\left(\mathscr{H}, \mathscr{H}^{\perp}\right)$ such that $\mathscr{H}_{t}$ is the graph of $\varphi(t)$ for $|t|<\varepsilon$; that is, $\mathscr{H}_{t}=\{\xi+\varphi(t) \xi: \xi \in \mathscr{H}\}$. Here $\mathscr{H}^{\perp}$ denotes the orthogonal complement of $\mathscr{H}=T_{e} H$ in $\mathscr{N}=T_{e} N$. Let $\xi$ be an arbitrary element of $\mathscr{H}=T_{e} H$, and let $\xi(t)=d L_{\gamma(t)^{-1}}\left(P_{t} \xi\right)$ in the notation of the lemma above. Then $\xi(t) \in \mathscr{H}_{t}$ for all $t$ by (a) of that result and we obtain

$$
\xi(t)=\bar{\xi}(t)+\varphi(t) \bar{\xi}(t) \quad \text { for some } C^{\infty} \text { curve } \bar{\xi}:(-\varepsilon, \varepsilon) \rightarrow \mathscr{H} .
$$

Now $\varphi(0)=0 \in \operatorname{Hom}\left(\mathscr{H}, \mathscr{H}^{\perp}\right)$ since $\mathscr{H}_{0}=\mathscr{H}$, and $\varphi^{\prime}(0)=0 \in \operatorname{Hom}\left(\mathscr{H}, \mathscr{H}^{\perp}\right)$ if and only if the curve $t \rightarrow \mathscr{H}_{t}=G(\gamma t)$ in $G(k, n)$ has zero velocity at $t=0$. If $d G\left(\xi^{*}\right)=0$, then $\varphi^{\prime}(0)=0$, and from $(*)$ we conclude that $\xi^{\prime}(0)=\bar{\xi}^{\prime}(0) \in \mathscr{H}$. On the other hand $\xi^{\prime}(0)=-\nabla_{\xi^{*}} \xi$ by (b) of the lemma above. Therefore $\mathscr{H}$ contains $\nabla_{\xi^{*}} \xi$ for all $\xi \in \mathscr{H}$ if $d G\left(\xi^{*}\right)=0$.

Conversely, if $\mathscr{H}$ contains $\nabla_{\xi^{*}} \xi$ for all $\xi \in \mathscr{H}$, then $\xi^{\prime}(0)=-\nabla_{\xi^{*}} \xi$ lies in $\mathscr{H}$ for all $\xi \in \mathscr{H}$ by (b) of the lemma above. It follows from $(*)$ above that $\mathscr{H}$ contains $\varphi^{\prime}(0) \xi=\xi^{\prime}(0)-\bar{\xi}^{\prime}(0)$ for all $\xi \in \mathscr{H}$ since $\bar{\xi}(0)=\xi$ and $\varphi(0)=0$. Since $\varphi^{\prime}(0) \in \operatorname{Hom}\left(\mathscr{H}, \mathscr{H}^{\perp}\right)$ we conclude that $\varphi^{\prime}(0) \xi \in \mathscr{H} \cap \mathscr{H}^{\perp}=\{0\}$ for all $\xi \in \mathscr{H}$. Hence $\varphi^{\prime}(0)=0$ and $d G\left(\xi^{*}\right)=0$.

We now prove the main result of this section.

(4.2) Theorem. Let $\{N,\langle\rangle$,$\} be a simply-connected, nonsingular, 2-step nilpo-$ tent Lie group of dimension $n \geq 3$ with a left invariant metric. Let $H$ be a totally 
geodesic submanifold of $N$ of dimension $k \geq 2$. Assume that the Gauss map $G: H \rightarrow G(k, n)$ fails to have maximal rank at some point $g$ of $H$. Then the Gauss map is constant on $H$, and $H$ is an open subset of $L_{g}(\bar{H})$, where $\bar{H}$ is a totally geodesic subgroup of $N$.

We first reduce to the case that $H$ contains the identity $e$ and $G: H \rightarrow$ $G(k, n)$ fails to have maximal rank at $e$. By hypothesis $G$ fails to have maximal rank at some point $g$ of $H$. If we define $H^{*}=L_{g^{-1}}(H)$, then $H^{*}$ is a $k$-dimensional, totally geodesic submanifold of $N$ that contains $e$. If $G^{*}: H^{*} \rightarrow G(k, n)$ is the Gauss map of $H^{*}$, then it is routine to check that $G^{*}\left(g^{*}\right)=G\left(g g^{*}\right)$ for all $g^{*} \in H^{*}$. Hence $G^{*}$ fails to have maximal rank at $e$. If (4.2) holds in the case that $g=e$, then $H^{*}$ is an open subset of a totally geodesic subgroup $\bar{H}$ of $N$. It follows that $H=L_{g}\left(H^{*}\right)$ is an open subset of $L_{g}(\bar{H})$.

We assume that $H$ contains $e$ and that the Gauss map $G: H \rightarrow G(k, n)$ fails to have maximal rank at $e$. It suffices to prove that $\mathscr{H}=T_{e} H$ is a totally geodesic subalgebra of $\mathscr{N}$. It will then follow from (3.11) that $H$ is an open subset of a totally geodesic subgroup $\bar{H}$ of $N$.

From (4.1) we obtain

(1) There exists a nonzero vector $\xi^{*}$ of $\mathscr{H}$ such that $\nabla_{\xi^{*}} \xi \in \mathscr{H}$ for every vector $\xi \in \mathscr{H}$.

Now write $\xi^{*}=X_{0}+Z_{0}$, where $\xi^{*}$ is the vector of $(1), X_{0} \in \mathscr{V}$ and $Z_{0} \in \mathscr{Z}$. If $X_{0}$ and $Z_{0}$ are both nonzero, then $\mathscr{H} \cap \mathscr{V}$ contains the nonzero vector $\nabla_{\xi^{*}} \xi^{*}=-j\left(Z_{0}\right) X_{0}$ by (1). If $X_{0}$ or $Z_{0}$ is zero, then $\mathscr{H} \cap \mathscr{Z}$ or $\mathscr{H} \cap \mathscr{V}$ is nonzero. Hence in all cases $(2.2)$ yields

(2) $\mathscr{H}=(\mathscr{H} \cap \mathscr{V}) \oplus(\mathscr{H} \cap \mathscr{Z})$ (orthogonal direct sum).

We consider separately the following cases: (1) $Z_{0} \neq 0$ and (2) $Z_{0}=0$ and $\xi^{*}=X_{0} \in \mathscr{H} \cap \mathscr{V}$. We begin with case (1). We may assume that in (2) above $\mathscr{H} \cap \mathscr{V} \neq\{0\}$ for otherwise $\mathscr{H} \subseteq \mathscr{Z}$ and $\mathscr{H}$ is an abelian, totally geodesic subalgebra of $\mathscr{N}$ by (1.10) or (3.7). Note that $X_{0} \in \mathscr{H} \cap \mathscr{V}$ and $0 \neq Z_{0} \in \mathscr{H} \cap \mathscr{Z}$ by (2). By (d) of (2.2) it suffices to prove that $j\left(Z_{0}\right)$ leaves $\mathscr{H} \cap \mathscr{V}$ invariant. To verify this let $X^{*} \in \mathscr{H} \cap \mathscr{V}$ be given arbitrarily. From (1) we know that $\mathscr{H}$ contains

$$
\nabla_{\xi^{*}} X^{*}=\nabla_{X_{0}} X^{*}+\nabla_{Z_{0}} X^{*}=\frac{1}{2}\left[X_{0}, X^{*}\right]-\frac{1}{2} j\left(Z_{0}\right) X^{*} .
$$

By (2) $\mathscr{H}$ contains the $\mathscr{V}$ and $\mathscr{Z}$ components of $\nabla_{\xi^{*}} X^{*}$, and in particular $\mathscr{H}$ contains $j\left(Z_{0}\right) X^{*}$.

We now consider case (2), where $\xi^{*}=X_{0} \in \mathscr{H} \cap \mathscr{V}$ and $\xi^{*}$ is the vector of (1) above. In the discussion that follows we do not assume that $\mathscr{H} \cap \mathscr{Z}$ is nonzero in the direct sum decomposition of (2) above. However, if $\mathscr{H} \subseteq \mathscr{V}$ then some of the assertions below are vacuously true. We proceed in several steps.

Step 1. Let $\mathscr{V}^{*}=\left\{X \in \mathscr{H} \cap \mathscr{V}: \nabla_{X} \xi \in \mathscr{H}\right.$ for all $\left.\xi \in \mathscr{H}\right\}$. Then $\mathscr{V}^{*}$ is a nonzero subspace of $\mathscr{H} \cap \mathscr{V}$ that is invariant under $j(Z)$ for all $Z \in \mathscr{H} \cap \mathscr{Z}$.

Clearly $\mathscr{V}^{*}$ is a subspace of $\mathscr{H} \cap \mathscr{V}$ and $\mathscr{V}^{*}$ is nonempty since it contains $\xi^{*}=X_{0}$. If $0 \neq X \in \mathscr{V}^{*}$ and $Z \in \mathscr{H} \cap \mathcal{Z}$ are given arbitrarily, then $\mathscr{H} \cap \mathscr{V}$ contains $-2 \nabla_{X} Z=j(Z) X$ by the definition of $\mathscr{V}^{*}$. We must show that $\nabla_{j(Z) X} \xi \in \mathscr{H}$ for all $\xi \in \mathscr{H}$ to conclude that $j(Z) X \in \mathscr{V}^{*}$. If $Y \in \mathscr{H} \cap$ $\mathscr{V}$ is arbitrary, then by (1.10), (1.11) and (2.1) $\mathscr{H}$ contains $2 R(Y, Z) X=$ 
$\frac{1}{2}[j(Z) X, Y]=\nabla_{j(Z) X} Y$. If $Z^{*} \in \mathscr{H} \cap \mathscr{Z}$ is arbitrary, then $\mathscr{H}$ contains $2 R\left(X, Z^{*}\right) Z=-\frac{1}{2} j\left(Z^{*}\right) j(Z) X=\nabla_{j(Z) X} Z^{*}$. Hence $\nabla_{j(Z) X} \xi \in \mathscr{C}$ for all $\xi \in \mathscr{H}$ by (2), and the proof of Step 1 is complete.

Step 2. If $\mathscr{V}^{* *}$ denotes the orthogonal complement in $\mathscr{H} \cap \mathscr{V}$ of $\mathscr{V}^{*}$, then $\left[\mathscr{V}^{*}, \mathscr{V}^{* *}\right]=\{0\}$.

Let $X^{*}=\mathscr{V}^{*}$ and $X^{* *} \in \mathscr{V}^{* *}$ be given, and let $Z=\left[X^{*}, X^{* *}\right]$. Note that $Z=2 \nabla_{X^{*}} X^{* *}$ lies in $\mathscr{H} \cap \mathscr{Z}$ since $X^{*} \in \mathscr{V}^{*}$. Hence $|Z|^{2}=\left\langle\left[X^{*}, X^{* *}\right], Z\right\rangle=$ $\left\langle j(Z) X^{*}, X^{* *}\right\rangle=0$ since $j(Z) X^{*} \in \mathscr{V}^{*}$ by Step 1.

Step 3. $\mathscr{H} \cap \mathscr{V}$ is invariant under $j(Z)$ for all $Z \in \mathscr{H} \cap \mathscr{Z}$.

Since $\mathscr{H} \cap \mathscr{V}=\mathscr{V}^{*} \oplus \mathscr{V}^{* *}$ it suffices by Step 1 to show that $j(Z) \mathscr{V}^{* *} \subseteq$ $\mathscr{H} \cap \mathscr{V}$ for all $Z \in \mathscr{H} \cap \mathscr{Z}$. Let $Z \in \mathscr{H} \cap \mathscr{Z}$ and $X^{* *} \in \mathscr{V}^{* *}$ be given. Fix a nonzero element $X$ of $\mathscr{V}^{*}$. From Lemma A of (1.12) and (2.1) we see that $R_{X}: \mathscr{H} \cap \mathscr{Z} \rightarrow \mathscr{H} \cap \mathscr{Z}$ is an isomorphism. By $(1.11) R_{X}\left(Z^{*}\right)=\frac{1}{4}\left[X, j\left(Z^{*}\right) X\right]$ for all $Z^{*} \in \mathscr{H} \cap \mathscr{Z}$. We conclude

There exists $Z^{*} \in \mathscr{H} \cap \mathscr{Z}$ such that $\left[X, j\left(Z^{*}\right) X\right]=Z$.

By Step $1, \mathscr{V}^{*}$ contains $j\left(Z^{*}\right) X$ and hence by (1.11), (2.1), Step 2 and $(*)$ we see that $\mathscr{H} \cap \mathscr{V}$ contains

$$
\begin{aligned}
2 R\left(X, j\left(Z^{*}\right) X\right) X^{* *}= & j\left(\left[X, j\left(Z^{*}\right) X\right]\right) X^{* *}-\frac{1}{2} j\left(\left[j\left(Z^{*}\right) X, X^{* *}\right]\right) X \\
& +\frac{1}{2} j\left(\left[X, X^{* *}\right]\right) j\left(Z^{*}\right) X=j(Z) X^{* *} .
\end{aligned}
$$

Step 4 (conclusion of case (2)). We consider separately the cases (1) $\mathscr{H} \cap \mathscr{Z} \neq$ $\{0\}$ and (2) $\mathscr{H} \subseteq \mathscr{V}$ (cf. (2) above). If (1) holds, then $\mathscr{H}$ is a totally geodesic subalgebra by Step 3 and (d) of (2.2). We assume that $\mathscr{H} \subseteq \mathscr{V}$. To show that $\mathscr{H}$ is a totally geodesic subalgebra of $\mathscr{N}$ it suffices by (3.7) to show that $\mathscr{H}$ is an abelian subspace of $\mathscr{V}$. We suppose that $\mathscr{H}$ is not abelian and obtain a contradiction. Let $Y_{1}, Y_{2}$ be elements of $\mathscr{H}$ such that $Z=\left[Y_{1}, Y_{2}\right] \neq 0$, and let $X$ be any nonzero element of $\mathscr{V}^{*} \subseteq \mathscr{H} \cap \mathscr{V}$. We observe first that $\left[X, Y_{1}\right]=\left[X, Y_{2}\right]=0$; since $X \in \mathscr{V}^{*}$ it follows that $\{0\}=\mathscr{H} \cap \mathscr{Z}$ contains $\nabla_{X} Y_{i}=\frac{1}{2}\left[X, Y_{i}\right]$ for $i=1,2$. Next, $\mathscr{H}$ contains

$$
2 R\left(Y_{1}, Y_{2}\right) X=j\left(\left[Y_{1}, Y_{2}\right]\right) X-\frac{1}{2} j\left(\left[Y_{2}, X\right]\right) Y_{1}+\frac{1}{2} j\left(\left[Y_{1}, X\right]\right) Y_{2}=j(Z) X .
$$

Therefore since $X \in \mathscr{V}^{*}$ we conclude that $\{0\}=\mathscr{H} \cap \mathscr{Z}$ contains $2 \nabla_{X} j(Z) X=$ $[X, j(Z) X]$. However, $[X, j(Z) X]$ cannot be zero since both $X$ and $Z$ are nonzero, which implies that $\langle[X, j(Z) X], Z\rangle=|j(Z) X|^{2}>0$. This contradiction shows that $\mathscr{H}$ is abelian if $\mathscr{H} \subseteq \mathscr{V}$ and completes the proof of (4.2).

(4.3) Corollary. Let $\{N,\langle\rangle$,$\} be a simply-connected, nonsingular, 2-step nil-$ potent Lie group of dimension $n \geq 3$ with a left invariant metric. Let $H$ be a totally geodesic submanifold of $N$ of dimension $k \geq 2$ that contains the identity $e$. Let $\mathscr{H}$ denote $T_{e} H$. Then the following are equivalent:

(1) $\mathscr{H}$ is a totally geodesic subalgebra of $\mathscr{N}$.

(2) $H$ is an open subset of a totally geodesic subgroup $\bar{H}$.

(3) $\mathscr{H}$ satisfies one of the following:

(a) $\mathscr{H}$ is an abelian subspace of $\mathscr{V}$.

(b) $\mathscr{H}$ is a subspace of $\mathscr{Z}$. 
(c) The subspaces $\mathscr{H} \cap \mathscr{V}$ and $\mathscr{H} \cap \mathscr{Z}$ are both nonzero and $\mathscr{H} \cap \mathscr{V}$ is invariant under $j(Z)$ for some nonzero element $Z$ of $\mathscr{H} \cap \mathscr{Z}$.

(d) The subspaces $\mathscr{H} \cap \mathscr{V}$ and $\mathscr{H} \cap \mathscr{Z}$ are both nonzero and ad $X(\mathscr{H} \cap \mathscr{V})$ $=\mathscr{H} \cap \mathscr{Z}$ for some nonzero element $X$ of $\mathscr{H} \cap \mathscr{V}$.

(4) The Gauss map $G: H \rightarrow G(k, n)$ fails to have maximal rank at some point $g$ of $H$.

Proof. The properties (1) and (2) are equivalent by (3.11). The assertion $(1) \Rightarrow(3)$ is contained in the statement of $(3.5)$. The assertion $(4) \Rightarrow(1)$ is a special case of $(4.2)$, and $(2) \Rightarrow(4)$ is obvious. It remains to prove $(3) \Rightarrow(1)$. If (3a) or (3b) holds, then (1) holds by (3.7). If (3c) holds, then (1) holds by (d) of (2.2).

Assume now that (3d) holds. To show that (1) holds it suffices to show that (4) holds. Let $X$ be a nonzero element of $\mathscr{H} \cap \mathscr{V}$ such that ad $X(\mathscr{H} \cap \mathscr{V})=\mathscr{H} \cap \mathscr{Z}$. We show first that $j(Z) X \in \mathscr{H} \cap \mathscr{V}$ for all $Z \in \mathscr{H} \cap \mathscr{Z}$. Let $Z \in \mathscr{H} \cap \mathscr{Z}$ be given and choose $Y \in \mathscr{H} \cap \mathscr{V}$ such that $[X, Y]=\operatorname{ad} X(Y)=Z$. Then $\mathscr{H}$ contains $\frac{4}{3} R(X, Y) X=j([X, Y]) X=j(Z) X$.

By (4.1) it suffices to show that $\nabla_{X} \xi \in \mathscr{H}$ for any element $\xi \in \mathscr{H}$, for then $d G(X)=0$ and $G: H \rightarrow G(k, n)$ does not have maximal rank at $e$. We note that $\mathscr{H}=\mathscr{H} \cap \mathscr{V} \oplus \mathscr{H} \cap \mathscr{Z}$ by (2.2) and the hypothesis of (3d). Let $\xi$ be any element of $\mathscr{H}$ and write $\xi=X^{*}+Z^{*}$, where $X^{*} \in \mathscr{H} \cap \mathscr{V}$ and $Z^{*} \in \mathscr{H} \cap \mathscr{Z}$. Now $\nabla_{X} \xi=\frac{1}{2}\left[X, X^{*}\right]-\frac{1}{2} j\left(Z^{*}\right) X=\frac{1}{2}$ ad $X\left(X^{*}\right)-\frac{1}{2} j\left(Z^{*}\right) X$. The first term lies in $\mathscr{H}$ since ad $H(\mathscr{H} \cap \mathscr{V})=\mathscr{H} \cap \mathscr{Z}$ while the second term lies in $\mathscr{H}$ by the previous paragraph. This completes the proof of $(3 \mathrm{~d}) \Rightarrow(4)$.

\section{WHEN TOTALLY GEODESIC SUBMANIFOLDS ARE SUBGROUPS}

In this section we look for conditions under which a totally geodesic submanifold $H$, not necessarily complete, must be an open subset of $L_{g}\left(H^{*}\right)$, where $g$ is a point of $H$ and $H^{*}$ is a totally geodesic subgroup of $N$. If $\operatorname{dim} H>\operatorname{dim} Z$, where $Z$ is the center of $N$, then this is always the case as we shall see below in (5.6). However, if $H$ has small dimension then one does not always have such a nice outcome. For example, if $H=\gamma$ is a geodesic of $N$ with $\gamma(0)=e$ and $\gamma^{\prime}(0)=\xi \in \mathscr{N}=T_{e} N$, then the 1-parameter subgroup $\{\exp (t \xi)\}$ is a geodesic of $N$ if and only if $\xi$ lies in $\mathscr{V}$ or $\mathscr{Z}$. See (2.11) of [E]. One may restate this fact by saying that for a 1-dimensional subspace $\mathscr{H}$ of $\mathscr{N}$, the manifold $H=\exp (\mathscr{H})$ is a geodesic of $N$ if and only if $\mathscr{H}$ is a totally geodesic subalgebra (see $(5.1)$ for a generalization). To verify this restatement one notes that if $X \in \mathscr{V}$ and $Z \in \mathscr{Z}$ are nonzero elements and if $\xi=X+Z$, then $\nabla_{\xi} \xi=-j(Z) X$ is not collinear with $\xi$. Hence a 1-dimensional subspace $\mathscr{H}$ of $\mathscr{N}$ is a totally geodesic subalgebra if and only if $\mathscr{H} \subseteq \mathscr{V}$ or $\mathscr{H} \subseteq \mathscr{Z}$.

As usual it suffices to consider totally geodesic submanifolds $H$ of $N$ that contain the identity. In this case one has better control if $\mathscr{H}=T_{e} H$ intersects either $\mathscr{V}$ or $\mathscr{Z}$ nontrivially (see (5.3)). Indeed if $N$ is of Heisenberg type, then $\mathscr{H}$ is a totally geodesic subalgebra if either $\mathscr{H} \cap \mathscr{V} \neq\{0\}$ or $\mathscr{H} \cap \mathscr{Z} \neq$ $\{0\}($ see $(5.14))$. Note that $\mathscr{H} \cap \mathscr{V} \neq\{0\}$ if $\operatorname{dim} H>\operatorname{dim} Z$. However, if $\operatorname{dim} H \leq \operatorname{dim} Z$, then we can say virtually nothing about $H$ with our present methods, which consist of a systematic use of the Gauss equations $(\S 2)$ and the Gauss map ( $§ 4)$.

We begin with a generalization of the remarks above about geodesics that are also 1-parameter subgroups. 
(5.1) Proposition. Let $\{N,\langle\rangle$,$\} be a simply-connected, nonsingular, 2-step$ nilpotent Lie group of dimension $n \geq 3$ with a left invariant metric. Let $\mathcal{N}$ denote the Lie algebra of $N$, and let $\mathscr{H}$ be a vector subspace of $\mathscr{N}$. Then $H=\exp (\mathscr{H})$ is a totally geodesic submanifold of $N$ if and only if $\mathscr{H}$ is a totally geodesic subalgebra of $\mathcal{N}$.

Proof. Clearly if $\mathscr{H}$ is a totally geodesic subalgebra of $\mathscr{N}$, then $H=\exp (\mathscr{H})$ is a totally geodesic subgroup of $N$. We suppose now that $H=\exp (\mathscr{H})$ is a totally geodesic submanifold of $N$, and prove that $\mathscr{H}$ is a totally geodesic subalgebra.

We shall need to review some facts about geodesics of $N$ that start at the identity. Let $\xi_{0}=X_{0}+Z_{0}$ be an arbitrary element of $\mathscr{N}$, where $X_{0} \in \mathscr{V}$ and $Z_{0} \in \mathscr{Z}$, and let $\gamma(t)$ be the geodesic of $N$ such that $\gamma(0)=e$ and $\gamma^{\prime}(0)=\xi_{0}$. We write $\gamma(t)=\exp (X(t)+Z(t))$, where $X(t) \in \mathscr{V}$ and $Z(t) \in \mathscr{Z}$ for every $t$. By Proposition (3.1) of [E] the functions $X(t), Z(t)$ satisfy the equations

$$
\begin{gathered}
X^{\prime \prime}(t)=j\left(Z_{0}\right) X^{\prime}(t) ; \quad X(0)=0, X^{\prime}(0)=X_{0}, \\
Z^{\prime}(t)+\frac{1}{2}\left[X^{\prime}(t), X(t)\right] \equiv Z_{0} ; \quad Z(0)=0, Z^{\prime}(0)=Z_{0} .
\end{gathered}
$$

Explicit formulae for $X(t)$ and $Z(t)$ are given in Proposition (3.5) of [E]. From these formulae it is easy to deduce the following facts:

(2) $|X(t)|$ is uniformly bounded above for all $t \in \mathbb{R}$.

(3) $Z(t)=t Z_{0}^{*}+B(t)$, where

(a) $|B(t)|$ is uniformly bounded above for all $t \in \mathbb{R}$,

(b) $Z_{0}^{*}=Z_{0}+Z_{0}^{\prime}$, where $Z_{0}^{\prime}$ is an element of $\mathscr{Z}$ such that $\left\langle Z_{0}^{\prime}, Z_{0}\right\rangle=$ $\frac{1}{2}\left|X_{0}\right|^{2}$.

We note that $H=\exp (\mathscr{H})$ is a closed subset of $N$ since exp: $N \rightarrow \mathscr{N}$ is a diffeomorphism. Hence $H$ is complete in the induced Riemannian metric. In particular if $\gamma$ is a geodesic of $N$ with $\gamma(0)=e$ and $\gamma^{\prime}(0) \in \mathscr{H}=T_{e} H$, then $\gamma(\mathbb{R}) \subseteq H$ since $H$ is totally geodesic.

We consider separately the cases (1) $\mathscr{H}$ is not contained in $\mathscr{V}$ and (2) $\mathscr{H}$ is contained in $\mathscr{V}$. We begin with case (1) and choose any vector $\xi_{0} \in \mathscr{H}$ such that $\xi_{0}=X_{0}+Z_{0}$, where $X_{0} \in \mathscr{V}, Z_{0} \in \mathscr{Z}$ and $Z_{0} \neq 0$. Let $\gamma$ be the geodesic of $N$ (and $H$ ) such that $\gamma(0)=e$ and $\gamma^{\prime}(0)=\xi_{0}$. If we write $\gamma(t)=\exp (X(t)+Z(t))$ as above, then $\mathscr{H}$ contains $X(t)+Z(t)$ for all $t$ since $\gamma(\mathbb{R}) \subseteq H=\exp (\mathscr{H})$. From (1) we conclude that $\mathscr{H}$ contains $X^{\prime \prime}(t)+Z^{\prime \prime}(t)=j\left(Z_{0}\right) X^{\prime}(t)-\frac{1}{2}\left[X^{\prime \prime}(t), X(t)\right]$ for all $t$, and in particular $\mathscr{H} \cap \mathscr{V}$ contains $j\left(Z_{0}\right) X_{0}=X^{\prime \prime}(0)+Z^{\prime \prime}(0)$. If $X_{0} \neq 0$, then $j\left(Z_{0}\right) X_{0} \neq 0$ while if $X_{0}=0$, then $\xi_{0}=Z_{0} \in \mathscr{H} \cap \mathscr{Z}$. In either case $\mathscr{H}=(\mathscr{H} \cap \mathscr{V}) \oplus(\mathscr{H} \cap \mathscr{Z})$ by (2.2) and this implies that $X_{0} \in \mathscr{H} \cap \mathscr{V}$ and $Z_{0} \in \mathscr{H} \cap \mathscr{Z}$. We have proved

(4) If $\mathscr{H}$ is not contained in $\mathscr{V}$, then $\mathscr{H}=(\mathscr{H} \cap \mathscr{V}) \oplus(\mathscr{H} \cap \mathscr{Z})$ and $\mathscr{H} \cap \mathscr{V}$ is invariant under $j\left(Z_{0}\right)$ for all $Z_{0} \in \mathscr{H} \cap \mathscr{Z}$.

By (4) and (d) of (2.2) it follows that $\mathscr{H}$ is a totally geodesic subalgebra of $\mathscr{N}$

We now consider case (2), where $\mathscr{H} \subseteq \mathscr{V}$. It suffices by (3.7) to prove that $\mathscr{H}$ is an abelian subspace of $\mathscr{V}$. We proceed by contradiction and assume that there exist elements $\xi, X_{0}$ in $\mathscr{H} \subseteq \mathscr{V}$ such that $Z_{0}=\frac{1}{2}\left[X_{0}, \xi\right] \neq 0$. Let

$$
\mathscr{H}_{\xi}=d L_{\exp (-\xi)}\left(T_{\exp (\xi)} H\right)=T_{e} H_{\xi},
$$


where $H_{\xi}=L_{\exp (-\xi)}(H)$. It follows immediately from (1.3) of [E] that

$$
\mathscr{H}_{\xi}=\left\{A+\frac{1}{2}[A, \xi]: A \in \mathscr{H}\right\} .
$$

If we define $\xi_{0}=X_{0}+Z_{0}$, then $\xi_{0} \in \mathscr{H}_{\xi}=T_{e} H_{\xi}$ by (5). The submanifold $H_{\xi}$ is complete and totally geodesic since $H=L_{\exp (\xi)}\left(H_{\xi}\right)$ has this property. Hence if $\gamma(t)$ is the unique geodesic in $N$ with $\gamma(0)=e$ and $\gamma^{\prime}(0)=\xi_{0}$, then $\gamma(\mathbb{R}) \subseteq \mathscr{H}_{\xi}$. If we write $\gamma(t)=\exp (X(t)+Z(t))$, where $X(t) \in \mathscr{V}$ and $Z(t) \in \mathscr{Z}$ for all $t$, then $\exp (\mathscr{H})=H=L_{\exp (\xi)}\left(H_{\xi}\right)$ contains

$$
\exp (\xi) \cdot \gamma(t)=\exp \left(\xi+X(t)+Z(t)+\frac{1}{2}[\xi, X(t)]\right)
$$

(cf. (1.9)). Hence $\xi+X(t)+Z(t)+\frac{1}{2}[\xi, X(t)] \in \mathscr{H} \subseteq \mathscr{V}$ for all $t$, and we conclude that $Z(t)+\frac{1}{2}[\xi, X(t)] \equiv 0$. Hence $|Z(t)|=\frac{1}{2}|[\xi, X(t)]|$ is uniformly bounded above in $t$ by (2), and by (3) the vector $Z_{0}^{*}$ that appears in (3) must be zero. However $\left\langle Z_{0}^{*}, Z_{0}\right\rangle=\frac{1}{2}\left|X_{0}\right|^{2}+\left|Z_{0}\right|^{2}$ by $(3 \mathrm{~b})$, and we conclude that $Z_{0}=0$, which contradicts the choice of $Z_{0}$. Therefore if $\mathscr{H} \subseteq \mathscr{V}$, then $\mathscr{H}$ is abelian. This completes the proof of (5.1).

For future reference we collect some notation that has already appeared and introduce some new notation.

(5.2) Notation. Let $H$ denote a connected, totally geodesic submanifold of $N$ of dimension $k \geq 2$. If $H$ contains the identity $e$, then $\mathscr{H}$ denotes $T_{e} H$. For an element $g$ of $H$ we let $\mathscr{H}_{g}$ denote $G(g)=d L_{g^{-1}}\left(T_{g} H\right)=T_{e} H_{g}$, where $G: H \rightarrow G(k, n)$ denotes the Gauss map of $H$ and $H_{g}=L_{g^{-1}}(H)$.

Well positioned totally geodesic submanifolds.

(5.3) Definition. Let $\{N,\langle\rangle$,$\} be a simply-connected, nonsingular, 2-step$ nilpotent Lie group of dimension $n \geq 3$ with a left invariant metric. Let $H$ be a connected, totally geodesic submanifold of $N$ of dimension $k \geq 2$. We say that $H$ is well positioned at a point $g$ of $H$ if either $\mathscr{H}_{g} \cap \mathscr{V} \neq\{0\}$ or $\mathscr{H}_{g} \cap \mathscr{Z} \neq\{0\}$.

(5.4) Remarks. (a) If we let $\mathscr{V}, \mathscr{Z}$ also denote the left invariant distributions in $T N$ determined by the subspaces $\mathscr{V}, \mathscr{Z}$ of $\mathscr{N}=T_{e} N$, then it is clear that $H$ is well positioned at $g$ if and only if either $T_{g} H \cap \mathscr{V}(g) \neq\{0\}$ or $T_{g} H \cap \mathscr{Z}(g) \neq\{0\}$.

(b) If $H$ is well positioned at $g$ in $H$, then by (2.2) we have $\mathscr{H}_{g}=\left(\mathscr{H}_{g} \cap\right.$ $\mathscr{V}) \oplus\left(\mathscr{H}_{g} \cap \mathscr{Z}\right)$ or equivalently $T_{g} H=\left\{T_{g} H \cap \mathscr{V}(g)\right\} \oplus\left\{T_{g} H \cap \mathscr{Z}(g)\right\}$. If $H$ is well positioned at $g$ in $H$, then the totally geodesic submanifold $L_{n}(H)$ is well positioned at $n g$ for every element $n$ of $N$.

(5.5) Theorem. Let $\{N,\langle\rangle$,$\} be a simply-connected, nonsingular, 2-step nilpo-$ tent Lie group of dimension $n \geq 3$ with a left invariant metric. Let $H$ be a connected, totally geodesic submanifold of $N$ of dimension $k \geq 2$, and assume that $H$ is well positioned at every point of an open subset $O$. If $g$ is any point of $O$, then $\mathscr{H}=G(g)$ is a totally geodesic subalgebra of $\mathscr{N}$, and $H$ is an open subset of $L_{g}(\bar{H})$, where $\bar{H}=\exp (\mathscr{H})$ is a totally geodesic subgroup of $N$.

Before beginning the proof we discuss three useful consequences. 
(5.6) Corollary. Let $\{N,\langle\rangle$,$\} be a simply-connected, nonsingular, 2-step nil-$ potent Lie group of dimension $n \geq 3$ with a left invariant metric. Let $H$ be a connected, totally geodesic submanifold of $N$ that contains $e$ and satisfies $\operatorname{dim} H>\operatorname{dim} Z$, where $Z$ denotes the center of $N$. Then $\mathscr{H}=T_{e} H$ is a totally geodesic subalgebra of $\mathscr{N}$, and $H$ is an open subset of the totally geodesic subgroup $\bar{H}=\exp (\mathscr{H})$.

Proof of (5.6). Since $\operatorname{dim} \mathscr{H}_{g}=\operatorname{dim} \mathscr{H}>\operatorname{dim} \mathscr{Z}$ for every $g$ of $H$ it follows that $\mathscr{H}_{g} \cap \mathscr{V} \neq\{0\}$ for every $g$ of $H$. Hence $H$ is well positioned at every point, and the result follows from (5.5).

(5.7) Corollary. Let $\{N,\langle\rangle$,$\} be a simply-connected, 2-step nilpotent Lie group$ with 1-dimensional center and with a left invariant metric.

(1) Let $H$ be a connected, totally geodesic submanifold of $N$ with dimension $\geq 2$ that contains $e$. Then $\mathscr{H}=T_{e} H$ is a totally geodesic subalgebra of $\mathscr{N}$, and $H$ is an open subset of the totally geodesic subgroup $\bar{H}=\exp (\mathscr{H})$.

(2) Let $Z$ be a nonzero vector of $\mathscr{Z}$. Then there is a one-one correspondence between nonabelian totally geodesic subalgebras of $\mathcal{N}$ and nonzero subspaces of $\mathscr{V}$ that are invariant under $j(Z)$. In particular every totally geodesic submanifold $H$ of $N$ that is not flat must have odd dimension.

Proof of (5.7). Assertion (1) of (5.7) will follow immediately from (5.6) once we show that $N$ is nonsingular. If ad $\xi: \mathcal{N} \rightarrow N$ is not surjective for some $\xi \in \mathscr{N}$, then ad $\xi=0$ and $\xi \in \mathscr{Z}$ since $\mathscr{Z}$ is 1-dimensional. Therefore $N$ is nonsingular.

We prove (2). If $\mathscr{H}$ is a nonabelian, totally geodesic subalgebra of $\mathscr{N}$, then $\operatorname{dim} \mathscr{H} \geq 2$ and $\mathscr{H} \cap \mathscr{V} \neq\{0\}$ since $\operatorname{dim} \mathscr{Z}=1$. Hence $\mathscr{H}=(\mathscr{H} \cap \mathscr{V}) \oplus$ $(\mathscr{H} \cap \mathscr{Z})$ by (2.2), and $\mathscr{H} \cap \mathscr{Z} \neq\{0\}$ by (3.5). If $Z$ spans $\mathscr{Z}=\mathscr{H} \cap \mathscr{Z}$, then $j(Z)$ leaves invariant $W=\mathscr{H} \cap \mathscr{V}$ by (3.5). Conversely, if $W$ is a nonzero subspace of $\mathscr{V}$ invariant under $j(Z)$, then $\mathscr{H}=W \oplus \mathscr{Z}$ is a subalgebra of $\mathcal{N}$ that is totally geodesic by (3.7) and nonabelian by (3.6).

If $H$ is a totally geodesic submanifold of $N$ that contains $e$ and is not flat, then $\mathscr{H}=T_{e} H$ is a totally geodesic subalgebra by (1) above. Moreover, $\mathscr{H}$ is not abelian by (3.6) and (1.11) since $H$ is not flat. The discussion above shows that $j(Z)$ leaves invariant $\mathscr{H} \cap \mathscr{V}$, and hence $\mathscr{H} \cap \mathscr{V}$ has even dimension since $j(Z)$ is skew symmetric and nonsingular. Therefore $\mathscr{H}$ and $H$ have odd dimension.

(5.8) Corollary. Let $\{N,\langle\rangle$,$\} be a simply-connected, nonsingular, 2-step nil-$ potent Lie group with a left invariant metric. Then $N$ admits no totally geodesic hypersurface $H$.

Proof of (5.8). We argue by contradiction and assume that $N$ does admit a totally geodesic hypersurface $H$. As usual it suffices to consider the case that $H$ contains the identity. Let $n, v, z$ be the dimensions of $\mathscr{N}, \mathscr{V}, \mathscr{Z}$ respectively. By (3) of (1.4) we have $n=v+z \geq 2 z+1$, and hence $\operatorname{dim} H=$ $n-1 \geq 2 z>z=\operatorname{dim} Z$. By (5.6) it follows that $\mathscr{H}=T_{e} H$ is a totally geodesic subalgebra of $\mathscr{N}$. In particular $\mathscr{H}=(\mathscr{H} \cap \mathscr{V}) \oplus(\mathscr{H} \cap \mathscr{Z})$ by (3.5).

Since $\mathscr{H}$ is a hyperplane in $\mathscr{N}$ either (a) $\mathscr{H} \cap \mathscr{V}=\mathscr{V}$ and $\mathscr{H} \cap \mathscr{Z}$ has codimension 1 in $\mathscr{Z}$ or (b) $\mathscr{H} \cap \mathscr{Z}=\mathscr{Z}$ and $\mathscr{H} \cap \mathscr{V}$ has codimension 1 in $\mathscr{V}$. Case (a) cannot occur since it would follow that $\mathscr{Z}=[\mathcal{N}, \mathscr{N}]=[\mathscr{V}, \mathscr{V}]=$ $[\mathscr{H} \cap \mathscr{V}, \mathscr{H} \cap \mathscr{V}] \subseteq \mathscr{Z}$. Assume that case (b) occurs and let $Z$ be a nonzero 
element of $\mathscr{Z} \subseteq \mathscr{H}$. Then $\mathscr{H} \cap \mathscr{V}$ is invariant under $j(Z)$ by (3.5), and it follows that the 1-dimensional orthogonal complement of $\mathscr{H} \cap \mathscr{V}$ in $\mathscr{V}$ is also invariant under $j(Z)$ since $j(Z)$ is skew symmetric. This is impossible since $j(Z)$ is also nonsingular and therefore has no eigenvectors in a real vector space. Hence case (b) does not arise either, and this contradiction completes the proof of (5.8).

Proof of (5.5). We now begin the proof of (5.5) and for convenience we consider first a special case.

(5.9) Lemma. let $\{N,\langle\rangle$,$\} be a simply simply-connected, nonsingular, 2-step$ nilpotent Lie group of dimension $n \geq 3$ with a left invariant metric. Let $H$ be a connected, totally geodesic submanifold of $N$ of dimension $k \geq 2$ that contains $e$. For each $g \in H$ let $\mathscr{H}_{g}=d L_{g-1}\left(T_{g} H\right) \subseteq T_{e} N=\mathscr{N}$. Assume that $H$ admits an open set $O$ containing $e$ such that either $\mathscr{H}_{g} \subseteq \mathscr{V}$ for all $g \in O$ or $\mathscr{H}_{g} \subseteq \mathscr{Z}$ for all $g \in O$. Then $\mathscr{H}=T_{e} H$ is a flat, totally geodesic subalgebra of $\mathscr{N}$, and $H$ is an open subset of the flat totally geodesic subgroup $\bar{H}=\exp (\mathscr{H})$.

Proof. If $\mathscr{H} \subseteq \mathscr{Z}$, then $\mathscr{H}$ is clearly a flat, totally geodesic subalgebra of $\mathscr{N}$ by (3.7), and $H$ is an open subset of $\bar{H}=\exp (\mathscr{H})$ by (3.11). It remains to consider the case that $\mathscr{H}_{g} \subseteq \mathscr{V}$ for all $g \in O$.

Let $\left\{\xi_{1}, \ldots, \xi_{k}\right\}$ be linearly independent $C^{\infty}$ vector fields on $O$ that are tangent to $H$. By hypothesis $X_{i}^{*}(g)=d L_{g^{-1}}\left(\xi_{i}(g)\right)$ lies in $\mathscr{V}$ for every $g$ in $O$ and $1 \leq i \leq k=\operatorname{dim} H$. Let $p=\operatorname{dim} \mathscr{V}$ and let $\left\{X_{1}, \ldots, X_{p}\right\}$ be a basis for $\mathscr{V}$ such that $X_{i}=X_{i}^{*}(e)=\xi_{i}(e)$ for $1 \leq i \leq k$. Write $X_{i}^{*}(g)=$ $\sum_{r=1}^{p} \alpha_{i r}(g) X_{r}$, where $\left\{\alpha_{i r}\right\}$ are $C^{\infty}$ functions on $O \subseteq H$ such that $\alpha_{i j}(e)=$ $\delta_{i j}$ if $1 \leq i, j \leq k$ and $\alpha_{i j}(e)=0$ otherwise. Letting $X_{r}$ also denote a left invariant vector field on $N$ for $1 \leq r \leq p$ we obtain

$$
\xi_{i}(g)=\sum_{r=1}^{p} \alpha_{i r}(g) X_{r}(g) \quad \text { for all } g \in O,
$$

where $\alpha_{i j}(e)=\delta_{i j}$ if $1 \leq i, j \leq k$ and $\alpha_{i j}(e)=0$ otherwise.

We compute

$$
\left[\xi_{i}, \xi_{j}\right]=W_{i j}(g)+\sum_{r, s=1}^{p} \alpha_{i r}(g) \alpha_{j s}(g)\left[X_{r}, X_{s}\right](g),
$$

where

$$
W_{i j}(g)=\sum_{s=1}^{p}\left[\xi_{i}\left(\alpha_{j s}\right)\right](g) X_{s}(g)-\sum_{r, s=1}^{p} \alpha_{j s}(g)\left[X_{s}\left(\alpha_{i r}\right)\right](g) X_{r}(g)
$$

lies in $\mathscr{V}$ for every $g$ in $O$. For $1 \leq i, j \leq k$ the vector field $\left[\xi_{i}, \xi_{j}\right]$ is tangent to $H$ since $\xi_{i}, \xi_{j}$ are tangent to $H$. In particular $\mathscr{H}=T_{e} H$ contains

$$
\begin{aligned}
{\left[\xi_{i}, \xi_{j}\right](e) } & =W_{i j}(e)+\sum_{r, s=1}^{p} \alpha_{i r}(e) \alpha_{j s}(e)\left[X_{r}, X_{s}\right](e) \\
& =\left[X_{i}, X_{j}\right]+W_{i j}(e)
\end{aligned}
$$

by $(*)$ above. Since $\mathscr{H} \subseteq \mathscr{V}$ by hypothesis and $W_{i j}(e) \in \mathscr{V}$ we conclude that $\left[X_{i}, X_{j}\right] \in \mathscr{V} \cap \mathscr{Z}=\{0\}$. Hence $\mathscr{H}$ is an abelian subalgebra of $\mathscr{V}$ since 
$\left\{X_{1}, \ldots, X_{k}\right\}$ is a basis for $\mathscr{H}$. Finally, $\mathscr{H}$ is totally geodesic by (3.7), and $H$ is an open subset of $\bar{H}=\exp (\mathscr{H})$ by (3.11). This completes the proof of (5.9).

In the statement of (5.5) it suffices to consider the case that $g=e$ and $H$ is well positioned at every point of an open connected subset $O$ that contains $e$. We shall show that $\mathscr{H}=T_{e} H$ is a totally geodesic subalgebra of $\mathscr{N}$, and $H$ is an open subset of the totally geodesic subgroup $\bar{H}=\exp (\mathscr{H})$.

We break the remainder of the proof of (5.5) into several steps.

Step 1 . The functions $g \rightarrow \operatorname{dim}\left(\mathscr{H}_{g} \cap \mathscr{V}\right)$ and $g \rightarrow \operatorname{dim}\left(\mathscr{H}_{g} \cap \mathscr{Z}\right)$ are constant in $O$.

Proof. By the remarks in (5.4) we have

(1) $\mathscr{H}_{g}=\left(\mathscr{H}_{g} \cap \mathscr{V}\right) \oplus\left(\mathscr{H}_{g} \cap \mathscr{Z}\right)$ for all $g \in O$.

Let $k=\operatorname{dim} H$ and $n=\operatorname{dim} N$. The continuity of the Gauss map $G: H \rightarrow$ $G(k, n)$ and a standard argument by contradiction show

(2) For every $g \in O$ there exists an open neighborhood $U \subseteq O$ of $g$ such that

$$
\begin{array}{ll}
\operatorname{dim}\left(\mathscr{H}_{g^{*}} \cap \mathscr{V}\right) \leq \operatorname{dim}\left(\mathscr{H}_{g} \cap \mathscr{V}\right) & \text { for all } g^{*} \in U, \\
\operatorname{dim}\left(\mathscr{H}_{g^{*}} \cap \mathscr{Z}\right) \leq \operatorname{dim}\left(\mathscr{H}_{g} \cap \mathscr{Z}\right) & \text { for all } g^{*} \in U .
\end{array}
$$

From (1) we see that $k=\operatorname{dim}\left(\mathscr{H}_{g^{*}} \cap \mathscr{V}\right)+\operatorname{dim}\left(\mathscr{H}_{g^{*}} \cap \mathscr{Z}\right)$ for all $g^{*} \in U$, and therefore the inequalities in (2) are equalities. It follows that the functions $g^{*} \rightarrow \operatorname{dim}\left(\mathscr{H}_{g^{*}} \cap \mathscr{V}\right)$ and $g^{*} \rightarrow \operatorname{dim}\left(\mathscr{H}_{g^{*}} \cap \mathscr{Z}\right)$ are constant in $U$. The proof of Step 1 is complete since $U$ is a neighborhood of an arbitrary point $g$ of $O$ and $O$ is connected.

In the remainder of the proof we assume

$$
\begin{gathered}
\mathscr{H}_{g}=\left(\mathscr{H}_{g} \cap \mathscr{V}\right) \oplus\left(\mathscr{H}_{g} \cap \mathscr{Z}\right) \quad \text { for every } g \in O, \\
\operatorname{dim}\left(\mathscr{H}_{g} \cap \mathscr{Z}\right)=q \geq 1 \quad \text { for every } g \in O, \\
\operatorname{dim}\left(\mathscr{H}_{g} \cap \mathscr{V}\right)=p \geq 1 \quad \text { for every } g \in O .
\end{gathered}
$$

This assumption is justified by Step 1, (5.9) and (5.4).

Step 2. Let $X, Y$ be nonzero elements of $\mathscr{H} \cap \mathscr{V}$ and let $Z$ be a nonzero element of $\mathscr{H} \cap \mathscr{Z}$. Let $\gamma(t)$ be the geodesic of $N$ with $\gamma(0)=e$ and $\gamma^{\prime}(0)=$ $X$. For small $t$ let $\mathscr{H}_{t}=d L_{\gamma(t)^{-1}}\left(T_{\gamma(t)} H\right)$. Then

(a) $[X, Y]+O(t) \in \mathscr{H}_{t} \cap \mathscr{Z}$ for all small $t$,

(b) $j(Z) X+O(t) \in \mathscr{H}_{t} \cap \mathscr{V}$ for all small $t$,

where $O(t)$ denotes a vector in $\mathcal{N}$ such that $|O(t)| \leq M|t|$ for small $t$ and some positive constant $M$.

Proof. As in the proof of $(4.1)$ we note that $\gamma(-\varepsilon, \varepsilon) \subseteq H$ if $\varepsilon>0$ is sufficiently small since $\gamma^{\prime}(0) \in \mathscr{H}=T_{e} H$ and $H$ is totally geodesic. In particular the definition of $\mathscr{H}_{t}$ makes sense for $|t|<\varepsilon$. Making $\varepsilon$ smaller if necessary it follows from $(*)$ above that there exists a nontrivial decomposition $\mathscr{H}_{t}=\left(\mathscr{H}_{t} \cap\right.$ $\mathscr{V}) \oplus\left(\mathscr{H}_{t} \cap \mathscr{Z}\right)$ for $|t|<\varepsilon$. Applying the lemma in the proof of (4.1) to the vector $\xi^{*}=X$ we see that for $|t|<\varepsilon, \mathscr{H}_{t}$ contains

(a) $Z-t\left(\nabla_{X} Z\right)+O\left(t^{2}\right)=Z+\frac{1}{2} t j(Z) X+O\left(t^{2}\right)$,

(b) $Y-t\left(\nabla_{X} Y\right)+O\left(t^{2}\right)=Y-\frac{1}{2} t[X, Y]+O\left(t^{2}\right)$. 
By $(*)$ above $\mathscr{H}_{t} \cap \mathscr{V}$ contains $\frac{1}{2} t j(Z) X+O\left(t^{2}\right)$, the $\mathscr{V}$-component of $(a)$, and $\mathscr{Z}_{t} \cap \mathscr{Z}$ contains $-\frac{1}{2} t[X, Y]+O\left(t^{2}\right)$, the $\mathscr{Z}$-component of $(\mathrm{b})$. This completes the proof of Step 2.

We now complete the proof of (5.5). Let $X, Y$ be nonzero elements of $\mathscr{H} \cap \mathscr{V}$, and let $Z$ be a nonzero element of $\mathscr{H} \cap \mathscr{Z}$. Let $\mathscr{H}_{t}$ be defined as in Step 2. It follows that $\mathscr{H}_{t}=G(\gamma t) \rightarrow G(e)=\mathscr{H}$ as $t \rightarrow 0$ by the continuity of the Gauss map. From Step 2 we conclude (a) $[X, Y] \in \mathscr{H} \cap \mathscr{Z}$ and (b) $j(Z) X \in \mathscr{H} \cap \mathscr{V}$. From (a) and the fact that $\mathscr{H}=(\mathscr{H} \cap \mathscr{V}) \oplus(\mathscr{H} \cap \mathscr{Z})$ it follows that $[\mathscr{H}, \mathscr{H}]=[\mathscr{H} \cap \mathscr{V}, \mathscr{H} \cap \mathscr{V}] \subseteq \mathscr{H} \cap \mathscr{Z}$. Hence $\mathscr{H}$ is a subalgebra, and it follows from (b) and (3.7) that $\mathscr{H}$ is totally geodesic. Finally by (3.11) $H$ is an open subset of $\bar{H}=\exp (\mathscr{H})$.

Totally geodesic submanifolds that are well positioned at one point. Let $H$ be a totally geodesic submanifold of $N$. We have seen in (5.5) that if $H$ is well positioned at every point of an open subset of $H$, then $H$ is an open subset of $L_{g}(\bar{H})$, where $g$ is a point of $H$ and $\bar{H}$ is a totally geodesic subgroup of $N$. In some cases one may draw the same conclusion if $H$ is assumed to be well positioned at only one point of $H$, which we may assume to be the identity without loss of generality. We consider this problem in the remainder of the section.

The following consequence of (5.8) will be useful.

(5.10) Lemma. Let $\{N,\langle\rangle$,$\} be a simply-connected, nonsingular, 2-step nilpo-$ tent Lie group with a left invariant metric. Let $H$ be a connected, totally geodesic submanifold of $N$ of dimension $\geq 2$ that contains the identity, and let $\mathscr{H}=T_{e} H$. Let $\mathscr{H}^{*}$ be a nonabelian, 3-dimensional, totally geodesic subalgebra of $\mathscr{N}$ such that $\operatorname{dim}\left(\mathscr{H} \cap \mathscr{H}^{*}\right) \geq 2$. Then $\mathscr{H}^{*} \subseteq \mathscr{H}$.

Proof. By (3.5) $\left\{\mathscr{C}^{*},\langle\rangle,\right\}$ is a nonsingular, 2-step nilpotent metric Lie algebra. Let $H^{*}$ denote the 3-dimensional, totally geodesic subgroup $\exp \left(\mathscr{H}^{*}\right)$. If $\mathscr{H}$ does not contain $\mathscr{H}^{*}$, then $\operatorname{dim}\left(\mathscr{H} \cap \mathscr{H}^{*}\right)=2$, and by (3.9) $H \cap H^{*}$ contains a 2-dimensional, totally geodesic submanifold $H_{\varepsilon}$ such that $T_{e}\left(H_{\varepsilon}\right)=\mathscr{H} \cap \mathscr{C}^{*}$. This contradicts (5.8) applied to $H^{*}$ and its hypersurface $H_{\varepsilon}$.

(5.11) Proposition. Let $\{N,\langle\rangle$,$\} be a simply-connected, nonsingular, 2-step$ nilpotent Lie group with a left invariant metric. Let $H$ be a connected, totally geodesic submanifold of $N$ of dimension $\geq 2$ that contains the identity $e$. Let $\mathscr{H}=T_{e} H$. If $\operatorname{dim}(\mathscr{H} \cap \mathscr{Z})=1$, then $\mathscr{H}$ is a totally geodesic subalgebra of $\mathscr{N}$ and $H$ is an open subset of the totally geodesic subgroup $\bar{H}=\exp (\mathscr{H})$.

Proof. Let $Z$ be a unit vector in $\mathscr{H} \cap \mathscr{Z}$ that spans $\mathscr{H} \cap \mathscr{Z}$. By (2.2) we can write $\mathscr{H}=(\mathscr{H} \cap \mathscr{V}) \oplus(\mathscr{H} \cap \mathscr{Z})$, where $\mathscr{H} \cap \mathscr{V}$ is invariant under $j(Z)^{2}$. Note that $j(Z)^{2}$ is a negative definite symmetric linear map on $\mathscr{V}$ by (1.4) since $\mathcal{N}$ is nonsingular and $j(Z)$ is skew symmetric.

We show first that $j(Z)$ leaves $\mathscr{H} \cap \mathscr{V}$ invariant. By the remarks above $\mathscr{H} \cap \mathscr{V}$ has a basis $\left\{X_{1}, \ldots, X_{k}\right\}$ that consists of eigenvectors of $j(Z)^{2}$. It suffices to show that $j(Z) X_{i} \in \mathscr{H} \cap \mathscr{V}$ for $1 \leq i \leq k$. Let an integer $i$ with $1 \leq i \leq k$ be given. By $(1.11)$ and (2.1) $\mathscr{H} \cap \mathscr{Z}$ contains $4 R\left(Z, X_{i}\right) X_{i}=$ $\left[X_{i}, j(Z) X_{i}\right]$, and hence $\left[X_{i}, j(Z) X_{i}\right]=c_{i} Z$ for some $c_{i} \in \mathbb{R}$ since $Z$ spans $\mathscr{H} \cap \mathscr{Z}$. If $\mathscr{H}_{i}=\operatorname{span}\left\{X_{i}, Z, j(Z) X_{i}\right\}$, then $\operatorname{span}\left\{X_{i}, Z\right\} \subseteq \mathscr{H} \cap \mathscr{H}_{i}$, and by (3.8) $\mathscr{H}_{i}$ is a nonabelian, 3-dimensional, totally geodesic subalgebra of $\mathscr{N}$. By (5.10) $\mathscr{H}_{i} \subseteq \mathscr{H}$, and hence $j(Z) X_{i} \in \mathscr{H} \cap \mathscr{V}$ for $1 \leq i \leq k$. 
By the previous paragraph and (d) of (2.2) it follows that $\mathscr{H}$ is a totally geodesic subalgebra. By (3.11) $H$ is an open subset of $\bar{H}=\exp (\mathscr{H})$.

(5.12) Proposition. Let $\{N,\langle\rangle$,$\} be a simply-connected, nonsingular, 2-step$ nilpotent Lie group with a left invariant metric, and let $H$ be a 3-dimensional totally geodesic submanifold that contains $e$. If $\mathscr{H} \cap \mathscr{Z} \neq\{0\}$, where $\mathscr{H}=$ $T_{e} H$, then $\mathscr{H}$ is a totally geodesic subalgebra and $H$ is an open subset of the totally geodesic subgroup $\bar{H}=\exp (\mathscr{H})$.

Proof. If $\operatorname{dim}(\mathscr{H} \cap \mathscr{Z})=1$, then the result follows from (5.11), and if $\operatorname{dim}(\mathscr{H} \cap \mathscr{Z})=3$, then $\mathscr{H} \subseteq \mathscr{Z}$ and the result follows from (3.7) and (3.11). It remains only to eliminate the case that $\operatorname{dim}(\mathscr{H} \cap \mathscr{Z})=2$. If this occurs, then $\operatorname{dim}(\mathscr{H} \cap \mathscr{V})=1$ since $\mathscr{H}=(\mathscr{H} \cap \mathscr{V}) \oplus(\mathscr{H} \cap \mathscr{Z})$ by (2.2). The proof of $(5.12)$ will now be completed by

(5.13) Lemma. Let $\{N,\langle\rangle$,$\} be a simply-connected, nonsingular, 2-step nilpo-$ tent Lie group with a left invariant metric, and let $H$ be a connected, totally geodesic submanifold of dimension $k \geq 2$ that contains the identity. If $\mathscr{H} \cap \mathscr{V} \neq$ $\{0\}$, then $\operatorname{dim}(\mathscr{H} \cap \mathscr{V}) \geq 2$.

Proof. We assume that $\operatorname{dim}(\mathscr{H} \cap \mathscr{V})=1$ and obtain a contradiction. By (2.2) $\mathscr{H}=(\mathscr{H} \cap \mathscr{V}) \oplus(\mathscr{H} \cap \mathscr{Z})$, and hence $\operatorname{dim}(\mathscr{H} \cap \mathscr{Z}) \geq 1$ since $\operatorname{dim} \mathscr{H} \geq 2$. Let $X$ be a nonzero vector of $\mathscr{H} \cap \mathscr{V}$, and consider the curvature transformation $R_{X}: \mathscr{N} \rightarrow \mathscr{N}$ given by $\xi \rightarrow R(\xi, X) X$. By (1.12) and (2.1) $R_{X}$ leaves $\mathscr{H} \cap \mathscr{Z}$ invariant, and hence $R_{X}$ has a nonzero eigenvector $Z$ in $\mathscr{H} \cap \mathscr{Z}$ since $R_{X}$ is symmetric. By (1.11) we obtain

(1) $[X, j(Z) X]=4 R_{X}(Z)=\lambda Z$ for some $\lambda \in \mathbb{R}$.

By (2.2) $\mathscr{H} \cap \mathscr{V}$ is invariant under $j(Z)^{2}$ and since $X$ spans $\mathscr{H} \cap \mathscr{V}$ we obtain

(2) $j(Z)^{2} X=\mu X$ for some $\mu \in \mathbb{R}$.

By (1), (2) and (3.8) it follows that $\mathscr{C}^{*}=\operatorname{span}\{X, Z, j(Z) X\}$ is a nonabelian, 3-dimensional, totally geodesic subalgebra of $\mathscr{N}$, and $\mathscr{H}^{*} \cap \mathscr{H}=\operatorname{span}\{X, Z\}$ since $\mathscr{H} \cap \mathscr{V}$ is 1-dimensional. It follows from (5.10) that $\mathscr{H}^{*} \subseteq \mathscr{H}$, but this is impossible since $\mathscr{H}^{*} \cap \mathscr{V}$ is 2-dimensional.

(5.14) Proposition. Let $\{N,\langle\rangle$,$\} be a simply-connected, 2-step nilpotent Lie$ group with a left invariant metric that is of Heisenberg type. Let $H$ be a connected, totally geodesic submanifold of $N$ of dimension $k \geq 2$ that contains the identity. If $\mathscr{H} \cap \mathscr{V} \neq\{0\}$ or $\mathscr{H} \cap \mathscr{Z} \neq\{0\}$, then $\mathscr{H}=T_{e} H$ is a totally geodesic subalgebra, and $H$ is an open subset of the totally geodesic subgroup $\bar{H}=\exp (\mathscr{H})$.

Proof. By (2.2) we have

$$
\mathscr{H}=(\mathscr{H} \cap \mathscr{V}) \oplus(\mathscr{H} \cap \mathscr{Z}) .
$$

We first show that $\mathscr{H}$ is a subalgebra. Let elements $X, Y \in \mathscr{H} \cap \mathscr{V}$ be given arbitrarily. To show that $\mathscr{H}$ is a subalgebra it suffices by $(*)$ to show that $Z=[X, Y] \in \mathscr{H}$, and we may assume that $Z$ is nonzero. Since $H$ is totally geodesic we know from (1.11) and (2.1) that $\mathscr{H}$ contains $R(X, Y) X=$ $\frac{3}{4} j(Z) X$. Moreover, the definition of Heisenberg type and Lemma B of (1.12) imply that (a) $j(Z)^{2} X$ is collinear with $X$ and (b) $[X, j(Z) X]$ is collinear with $Z$. If $\mathscr{C}^{*}=\operatorname{span}\{X, Z, j(Z) X\}$, then (a), (b) and (3.8) imply that $\mathscr{H}^{*}$ is a nonabelian, 3-dimensional totally geodesic subalgebra of $\mathscr{N}$. Clearly 
$\operatorname{span}\{X, j(Z) X\} \subseteq \mathscr{H} \cap \mathscr{H}^{*}$, and hence by $(5.10) \mathscr{H}^{*} \subseteq \mathscr{H}$, which implies that $Z=[X, Y]$ lies in $\mathscr{H}$.

We have shown that $\mathscr{H}$ is a subalgebra of $\mathscr{N}$ and we now show that $\mathscr{H}$ is totally geodesic. The final assertion of (5.14) then follows from (3.11). If $\mathscr{H} \subseteq \mathscr{V}$, then $[\mathscr{H}, \mathscr{H}] \subseteq \mathscr{H} \cap \mathscr{Z}=\{0\}$ and $\mathscr{H}$ is abelian. By (3.7) it follows that $\mathscr{H}$ is totally geodesic. If $\mathscr{H} \subseteq \mathscr{Z}$, then $\mathscr{H}$ is also totally geodesic by (3.7). By $(*)$ it remains to consider the case that both $\mathscr{H} \cap \mathscr{V}$ and $\mathscr{H} \cap \mathscr{Z}$ are nonzero. Let $X, Z$ be nonzero vectors in $\mathscr{H} \cap \mathscr{V}, \mathscr{H} \cap \mathscr{Z}$ respectively. By (3.7) it suffices to prove that $j(Z) X \in \mathscr{H} \cap \mathscr{V}$. If $\mathscr{H}^{*}=\operatorname{span}\{X, Z, j(Z) X\}$, then as we explained earlier in the proof $\mathscr{H}^{*}$ is a nonabelian, 3-dimensional, totally geodesic subalgebra of $\mathscr{N}$. Since $\operatorname{span}\{X, Z\} \subseteq \mathscr{H} \cap \mathscr{H}^{*}$ it follows from (5.10) that $\mathscr{H}^{*} \subseteq \mathscr{H}$, and in particular $j(Z) X \in \mathscr{H} \cap \mathscr{V}$.

\section{A CHARACTERIZATION OF GROUPS OF HEISENBERG TYPE}

Let $\{N,\langle\rangle$,$\} be a simply-connected, nonsingular, 2-step nilpotent Lie group$ with a left invariant metric. If $N$ is of Heisenberg type, then every geodesic $\gamma$ of $N$ is contained in a 3-dimensional, totally geodesic submanifold $H$. To see this it suffices to consider the case that $\gamma(0)=e$ and $\gamma^{\prime}(0)=X+Z$, where $X \in \mathscr{V}$ and $Z \in \mathscr{Z}$. By (3.8) and (b) of Lemma B of (1.12) it follows that $\mathscr{H}=\operatorname{span}\{X, Z, j(Z) X\}$ is a totally geodesic subalgebra of $\mathscr{N}$. Hence $H=\exp (\mathscr{H})$ is a 3-dimensional, totally geodesic subgroup that contains $\gamma$ since $\gamma^{\prime}(0) \in T_{e} H=\mathscr{H}$.

In this section we prove a converse to the observation above.

(6.1) Theorem. Let $\{N,\langle\rangle$,$\} be a simply-connected, nonsingular, 2-step nilpo-$ tent Lie group with a left invariant metric. Assume that for every geodesic $\gamma$ of $N$ with $\gamma(0)=e$ there exists a connected, 3-dimensional, totally geodesic submanifold $H$ such that $\gamma^{\prime}(0) \in \mathscr{H}=T_{e} H$ and $\mathscr{H} \cap \mathscr{Z} \neq\{0\}$. Then $N$ is of Heisenberg type.

The hypothesis that $\mathscr{H} \cap \mathscr{Z}$ be nonzero in the statement above may be unnecessary, but we are able to avoid it only if $N$ has 1-dimensional center.

(6.2) Theorem. Let $\{N,\langle\rangle$,$\} be a simply-connected, nonsingular, 2-step nilpo-$ tent Lie group with a left invariant metric and a 1-dimensional center. Assume that for every geodesic $\gamma$ of $N$ with $\gamma(0)=e$ there exists a connected, 3dimensional, totally geodesic submanifold $H$ such that $\gamma^{\prime}(0) \in \mathscr{H}=T_{e} H$. Then for some positive number $\lambda$ and some integer $n \geq 1$, the group $\{N, \lambda\langle\rangle$, is isometric to a standard $(2 n+1)$-dimensional metric Heisenberg group.

Before proving (6.1) or (6.2) we need the following preliminary result.

(6.3) Lemma. Let $\{N,\langle\rangle$,$\} be a simply-connected, nonsingular, 2-step nilpo-$ tent Lie group with a left invariant metric. Assume that for every geodesic $\gamma$ of $N$ with $\gamma(0)=e$ there exists a 3-dimensional, totally geodesic subalgebra $\mathscr{H}$ such that $\gamma^{\prime}(0) \in \mathscr{H}$. Then $N$ is of Heisenberg type.

We break the proof into several steps.

Step 1 . Let $X, Z$ be arbitrary nonzero elements of $\mathscr{V}, \mathscr{Z}$. Then

(a) $\operatorname{span}\{X, Z, j(Z) X\}$ is a totally geodesic subalgebra of $\mathscr{N}$,

(b) $[X, j(Z) X]=c Z$ for some $c \in \mathbb{R}$, 
(c) $j(Z)^{2}=-\lambda(Z)$ Id for some positive number $\lambda(Z)$.

Proof. Let $\gamma$ be the geodesic of $N$ such that $\gamma(0)=e$ and $\gamma^{\prime}(0)=X+Z$. By hypothesis there exists a 3-dimensional, totally geodesic subalgebra $\mathscr{H}$ such that $\mathscr{H}$ contains $X+Z$. It follows from (3.5) that $\mathscr{H}=(\mathscr{H} \cap \mathscr{V}) \oplus(\mathscr{H} \cap \mathscr{Z})$, and hence both $X$ and $Z$ lie in $\mathscr{H}$. By (3.6) $\mathscr{H}$ is nonabelian and by (3.5) $\mathscr{H} \cap \mathscr{V}$ contains $j(Z) X$. Therefore $\mathscr{H}=\operatorname{span}\{X, Z, j(Z) X\}$ since $\mathscr{H}$ is 3-dimensional and $X, j(Z) X$ are nonzero orthogonal vectors in $\mathscr{H} \cap \mathscr{V}$. This proves (a). Assertion (b) follows since $\mathscr{H}$ is a subalgebra of $\mathscr{N}$, and $\mathscr{H} \cap \mathscr{Z}$ is a 1-dimensional subspace of $\mathscr{N}$ spanned by $Z$.

We prove (c). Observe that $j(Z)^{2}$ is negative definite and symmetric since $j(Z)$ is nonsingular and skew symmetric. It suffices to prove that $X$ is an eigenvector of $j(Z)^{2}$ since $X \in \mathscr{V}$ is arbitrary. The 2-dimensional vector space $\mathscr{H} \cap \mathscr{V}$ contains $X, j(Z) X$ and $j(Z)^{2} X$ since $\mathscr{H} \cap \mathscr{V}$ is invariant under $j(Z)$. Hence $X$ and $j(Z)^{2} X$ are collinear since both vectors are orthogonal to $j(Z) X$.

Step 2. If $Z, Z^{*}$ are arbitrary elements of $\mathscr{Z}$, then $j(Z) j\left(Z^{*}\right)+j\left(Z^{*}\right) j(Z)$ $=-g\left(Z, Z^{*}\right)$ Id, where $g$ is some positive definite bilinear form on $\mathscr{Z}$.

Proof. By (c) of Step $1, j(Z)^{2}, j\left(Z^{*}\right)^{2}$ and $j\left(Z+Z^{*}\right)^{2}=\left\{j(Z)+j\left(Z^{*}\right)\right\}^{2}$ are multiples of the identity. By expanding and simplifying $\left\{j(Z)+j\left(Z^{*}\right)\right\}^{2}$ it follows that $j(Z) j\left(Z^{*}\right)+j\left(Z^{*}\right) j(Z)=-g\left(Z, Z^{*}\right)$ Id for some function $g: \mathscr{Z} \times$ $\mathscr{Z} \rightarrow \mathbb{R}$. The function $g$ is clearly symmetric and is bilinear by the linearity of the map $Z \rightarrow j(Z), Z \in \mathscr{Z}$. Setting $Z=Z^{*}$ we find that $-2 j(Z)^{2}=$ $g(Z, Z)$ Id , which proves that $g(Z, Z)>0$ if $Z \neq 0$ since $j(Z)^{2}$ is negative definite.

We now complete the proof of (6.3). Let $g$ be the positive definite bilinear form on $\mathscr{Z}$ from Step 2, and let $S: \mathscr{Z} \rightarrow \mathscr{Z}$ be the positive definite symmetric linear map such that $g\left(Z, Z^{*}\right)=\left\langle S(Z), Z^{*}\right\rangle$ for all $Z, Z^{*} \in \mathscr{Z}$. Here $\langle$,$\rangle denotes the restriction to \mathscr{Z}$ of the given inner product on $\mathscr{N}$. Since $j(Z)^{2}=-\frac{1}{2} g(Z, Z) \operatorname{Id}=-\frac{1}{2}\langle S(Z), Z\rangle$ Id for all $Z \in \mathscr{Z}$ it suffices to show that $S$ is a positive multiple of the identity.

Let $\left\{Z_{1}, \ldots, Z_{m}\right\}$ be an orthonormal basis of $\mathscr{Z}$ consisting of eigenvectors of $S$, and let $\lambda_{1}, \ldots, \lambda_{m}$ be positive numbers such that $S\left(Z_{i}\right)=\lambda_{i} Z_{i}$ for every $i$. If $X, Z$ are any nonzero elements of $\mathscr{V}, \mathscr{Z}$, then by step 2 we have

$$
\begin{aligned}
{[X, j(Z) X] } & =\sum_{i=1}^{m}\left\langle[X, j(Z) X], Z_{i}\right\rangle Z_{i}=\frac{1}{2} \sum_{i=1}^{m} 2\left\langle j\left(Z_{i}\right) X, j(Z) X\right\rangle Z_{i} \\
& =-\frac{1}{2} \sum_{i=1}^{m}\left\{\left\langle X, j\left(Z_{i}\right) j(Z) X\right\rangle+\left\langle X, j(Z) j\left(Z_{i}\right) X\right\rangle\right\} Z_{i} \\
& =\frac{1}{2} \sum_{i=1}^{m} g\left(Z_{i}, Z\right)|X|^{2} Z_{i}=\left(\frac{1}{2}|X|^{2}\right) \sum_{i=1}^{m}\left\langle S\left(Z_{i}\right), Z\right\rangle Z_{i} \\
& =\left(\frac{1}{2}|X|^{2}\right) \sum_{i=1}^{m} \lambda_{i}\left\langle Z_{i}, Z\right\rangle Z_{i} .
\end{aligned}
$$

On the other hand by (b) of Step 1 there exists a constant $c$ such that $[X, j(Z) X]=c Z=\sum_{i=1}^{m} c\left\langle Z_{i}, Z\right\rangle Z_{i}$. If we choose $Z \in \mathscr{Z}$ so that $\left\langle Z_{i}, Z\right\rangle$ 
is nonzero for every $i$, then by equating the $Z_{i}$ components in the two expressions above for $[X, j(Z) X]$ we conclude that $\lambda_{i}=\left(2 c /|X|^{2}\right)$ for every $i$. Hence $S$ is a multiple of the identity, and the proof of $(6.3)$ is complete.

We now prove (6.1) and (6.2). Theorem (6.1) follows immediately from (6.3) and (5.12). We prove (6.2). Recall that $N$ is nonsingular by the proof of (5.7). Let $\gamma$ and $H$ be as in the statement of (6.2). By (5.7) $\mathscr{H}=T_{e} H$ is a totally geodesic subalgebra since $\operatorname{dim} \mathscr{Z}=1$, and hence $N$ is of Heisenberg type by (6.3). The discussion in (1.7) now shows that $\{N, \lambda\langle\rangle$,$\} is isometric$ to a standard metric $(2 n+1)$-dimensional Heisenberg group for some positive number $\lambda$ and some integer $n \geq 1$.

\section{REFERENCES}

[E] P. Eberlein, Geometry of 2-step nilpotent groups with a left invariant metric, Ann. Sci. Ecole Norm. Sup. (to appear).

[CDKR] M. Cowling, A. Dooley, A. Koranyi, and F. Ricci, H-type groups and Iwasawa decompositions, Adv. Math. 87 (1991), 1-41.

[K1] A. Kaplan, Riemannian nilmanifolds attached to Clifford modules, Geom. Dedicata 11 (1981), 127-136.

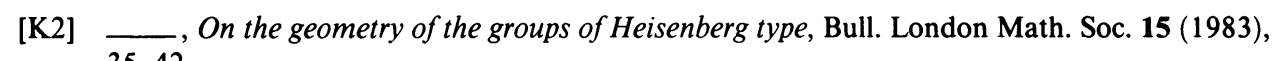
$35-42$.

[Ko] A. Koranyi, Geometric properties of Heisenberg type groups, Adv. Math. 56 (1985), 28-38.

Department of Mathematics, University of North Carolina, Chapel Hill, North CarOLINA 27599

E-mail address: pbe@math.unc.edu 